\title{
CD4 T follicular helper cell dynamics during SIV infection
}

\author{
Constantinos Petrovas, ${ }^{1}$ Takuya Yamamoto, ${ }^{1}$ Michael Y. Gerner, ${ }^{2}$ Kristin L. Boswell,, ${ }^{1}$ Kaska Wloka, \\ Emily C. Smith, ${ }^{1}$ David R. Ambrozak, ${ }^{1}$ Netanya G. Sandler, ${ }^{3}$ Katherina J. Timmer, ${ }^{3}$ Xiaoyong Sun, ${ }^{4}$ \\ Li Pan, ${ }^{4}$ Amanda Poholek, ${ }^{2}$ Srinivas S. Rao, ${ }^{5}$ Jason M. Brenchley, ${ }^{6}$ S. Munir Alam, ${ }^{7}$ \\ Georgia D. Tomaras, ${ }^{7}$ Mario Roederer, ${ }^{8}$ Daniel C. Douek, ${ }^{3}$ Robert A. Seder, ${ }^{9}$ \\ Ronald N. Germain, ${ }^{2}$ Elias K. Haddad, ${ }^{4}$ and Richard A. Koup'

\begin{abstract}
${ }^{1}$ Immunology Laboratory, Vaccine Research Center, ${ }^{2}$ Laboratory of Systems Biology, Lymphocyte Biology Section, and ${ }^{3}$ Human Immunology Section, Vaccine Research Center, National Institute of Allergy and Infectious Diseases (NIAID), NIH, Bethesda, Maryland, USA.

${ }^{4}$ VGTI Florida, Port St. Lucie, Florida, USA. ${ }^{5}$ Laboratory Animal Medicine, Vaccine Research Center, and ${ }^{6}$ Laboratory of Molecular Microbiology, NIAID, NIH, Bethesda, Maryland, USA. ${ }^{7}$ Duke Human Vaccine Institute, Duke University Medical School, Durham, North Carolina, USA. ${ }^{8}$ ImmunoTechnology Section and ${ }^{9}$ Cellular Immunology Section, Vaccine Research Center, NIAID, NIH, Bethesda, Maryland, USA.
\end{abstract}

\begin{abstract}
CD4 $\mathrm{T}$ follicular helper (TFH) cells interact with and stimulate the generation of antigen-specific B cells. TFH cell interaction with B cells correlates with production of SIV-specific immunoglobulins. However, the fate of TFH cells and their participation in SIV-induced antibody production is not well understood. We investigated the phenotype, function, location, and molecular signature of TFH cells in rhesus macaques. Similar to their human counterparts, TFH cells in rhesus macaques represented a heterogeneous population with respect to cytokine function. In a highly differentiated subpopulation of TFH cells, characterized by CD150 ${ }^{\text {lo }}$ expression, production of Th1 cytokines was compromised while IL-4 production was augmented, and cells exhibited decreased survival, cycling, and trafficking capacity. TFH cells exhibited a distinct gene profile that was markedly altered by SIV infection. TFH cells were infected by SIV; yet, in some animals, these cells actually accumulated during chronic SIV infection. Generalized immune activation and increased IL-6 production helped drive TFH differentiation during SIV infection. Accumulation of TFH cells was associated with increased frequency of activated germinal center $B$ cells and SIV-specific antibodies. Therefore, chronic SIV does not disturb the ability of TFH cells to help B cell maturation and production of SIV-specific immunoglobulins.
\end{abstract}

\section{Introduction}

The generation of antigen-specific B cells is dependent upon their interaction with $\mathrm{T}$ follicular helper (TFH) cells in the B cell follicles of the LN and spleen (SP) $(1,2)$. This interaction promotes the survival, isotype switching, and selection of high-affinity memory B cells and plasma cells ( 3 ) and is mediated by costimulatory molecules and soluble factors, such as IL-4, IL-10, and IL-21 $(1,4,5)$. TFH cells are characterized by high expression of CXCR5, BCL-6, programmed death-1 (PD-1), and ICOS $(1,5-8)$, and their function and trafficking is influenced by the availability of antigen and antigen-presenting cells $(5,9,10)$ and the expression of chemokine (11) and other receptors, including ICOS (12) and SLAM-family members $(13,14)$.

Previous RNA expression studies have shown that human TFH cells express a profile distinct from Th1 or Th2 cells $(15,16)$. A subpopulation of CXCR5 $5^{\text {hi }}$ TFH (germinal center [GC] TFH) cells expresses CD57 and has the ability to produce CXCL-13, the ligand of CXCR5 (17). Phenotypically, human CXCR5 hi TFH cells are characterized by high expression of CXCR4, CD95, SLAMassociated protein (SAP), CD154, BTLA, ICOS, and CD69 and dim expression of CD150 (also known as SLAM) (18).

Recent studies have shown that TFH cells can be generated from Th1 (19), Th2 (20), or other CD4 T cell lineages (21). All of these studies suggest that the in vivo origin of TFH cells is flexible when compared with that of other lineages. It is well established, however, that expression of BCL-6 as well as the function of soluble

Conflict of interest: The authors have declared that no conflict of interest exists. Citation for this article: J Clin Invest. 2012;122(9):3281-3294. doi:10.1172/JCI63039. factors like IL-6 $(21,22)$ is central to the lineage commitment of TFH cells $(7,8)$. In contrast, much less is known about the fate of TFH cells. It has been proposed that TFH cells can revert to a central memory (CM) phenotype or undergo cell death after the effector phase of a specific immune response (23). More recently, altered dynamics of TFH and B cells during chronic SIV infection were shown to correlate with higher titers of SIV-specific immunoglobulins (24).

Here, we investigated the TFH cell populations in rhesus macaques (RM). We found that RM TFH cells share many phenotypic characteristics with their human counterparts. We found that TFH cells often accumulate in LNs during SIV infection, and we identified the potential role of immune activation and IL- 6 production in driving that accumulation of TFH cells. Furthermore, the accumulation of TFH cells was associated with an expansion of the GC B cell compartment and increased circulating titers of SIV-specific antibodies.

\section{Results}

Phenotype of CD4 TFH cells in RMs. Expression of BCL-6 in CD4 T cells within GCs defines TFH cells (7). We therefore assessed BCL-6 expression in association with 2 other markers of TFH cells, PD-1 and $\mathrm{CXCR} 5$, in $\mathrm{CD}^{+}{ }^{+} \mathrm{CD} 4{ }^{+}$cells from RM LNs (Figure 1A). While high BCL-6 expression was found exclusively within the PD-1 ${ }^{\text {hi }}$ population of CD4 $\mathrm{T}$ cells, it was associated with CD4 T cells that expressed both intermediate and high levels of CXCR5 (Figure 1A). Consistent with GC localization, the PD- $1^{\text {hi }}$ cells had low expression of CCR7. In contrast, the vast majority of the BCL- $6^{\text {hi }}$ CXCR5 ${ }^{\text {interm/hi }}$ CD4 $\mathrm{T}$ cells expressed high levels of CCR7, a 
A $\mathrm{LN}: \mathrm{CD}^{+}{ }^{+} \mathrm{CD} 4{ }^{+} \mathrm{T}$ cells
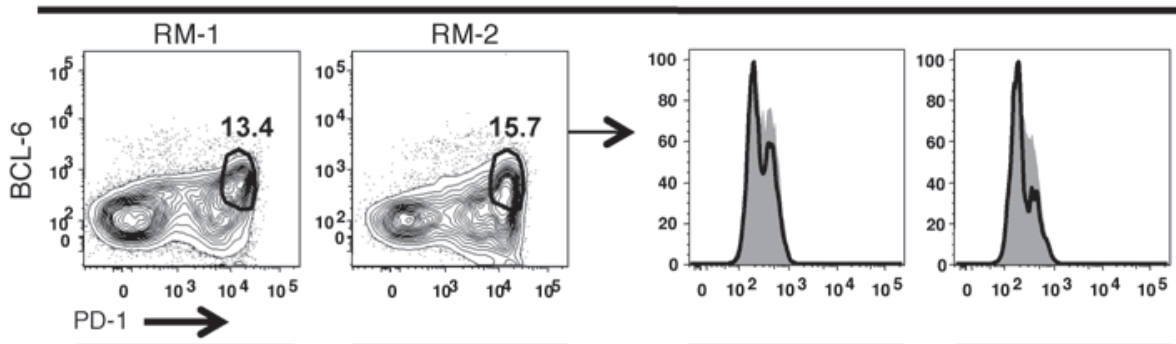

Gray: CM

Black line: Bcl-6hipD-1 ${ }^{\text {n }}$
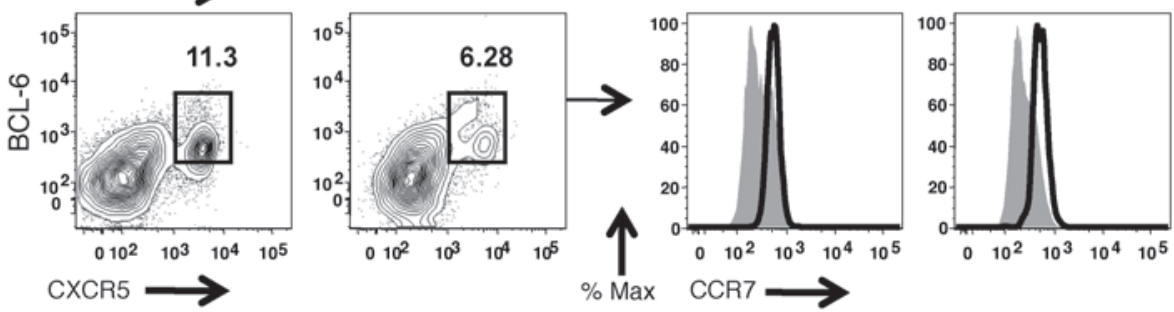

Gray: CM

Black line: $\mathrm{BCl}-6^{\text {hin }} \mathrm{CXCR} 5^{\text {dimn }}$

B
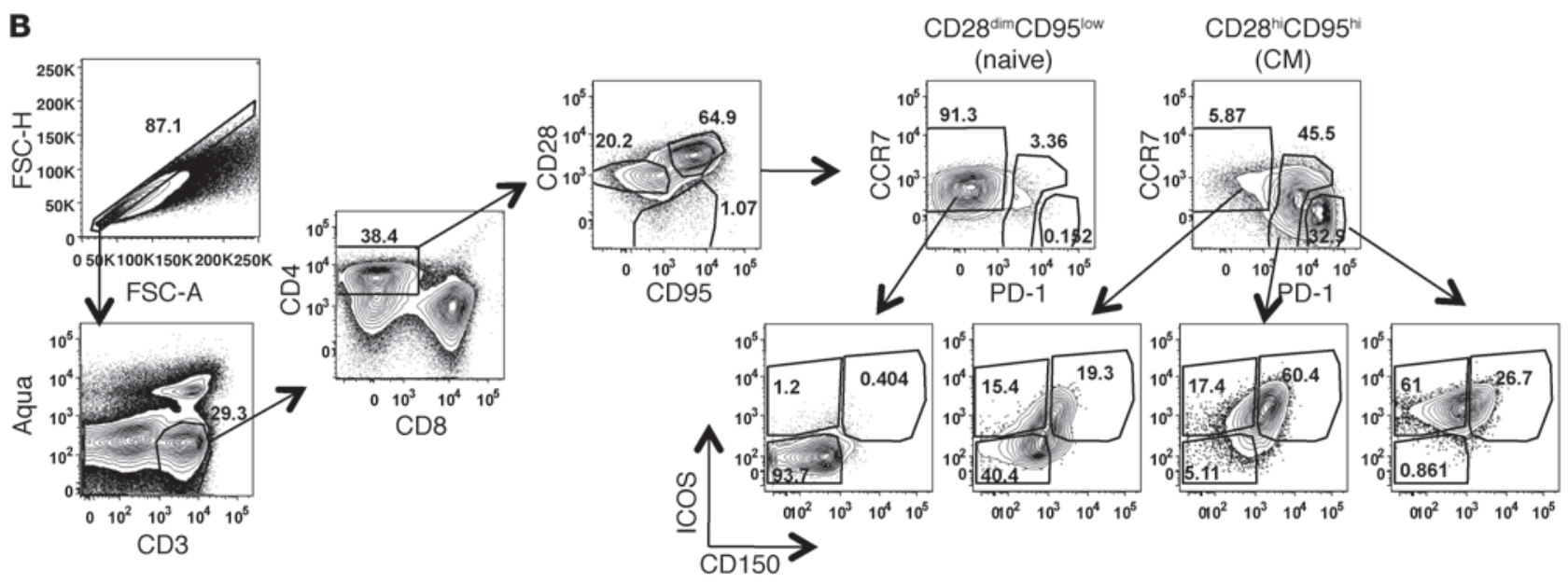

C

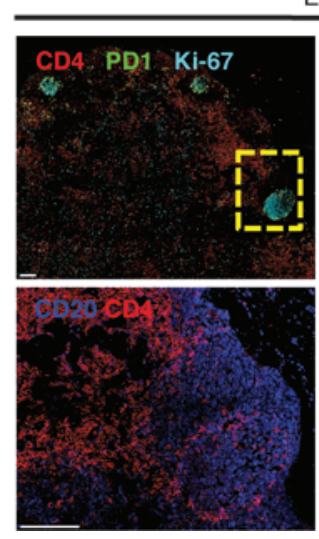

LN

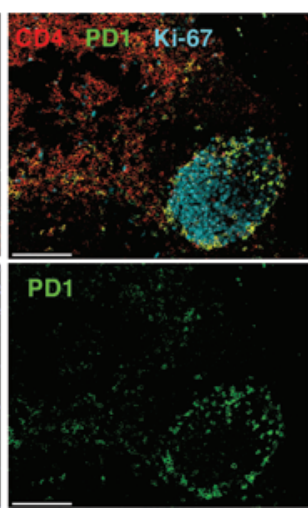

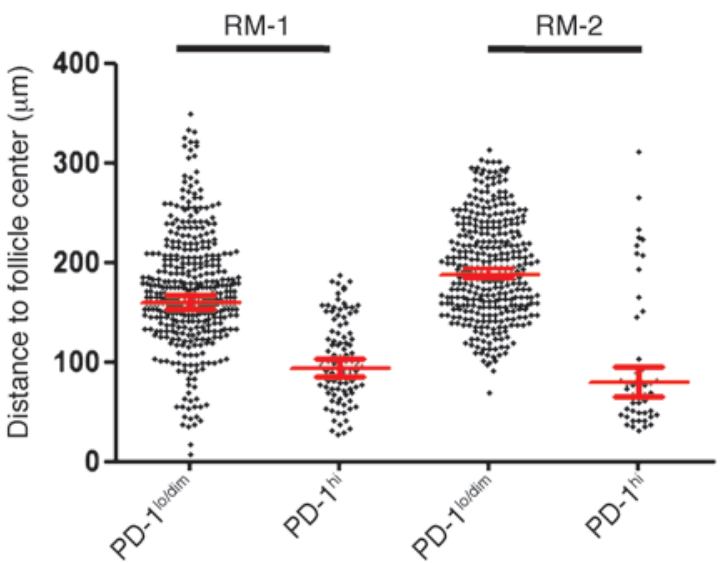

Figure 1

Characterization of TFH cells in RM LNs. (A) Flow cytometry contour plots, showing the expression of BCL-6 against either PD-1 or CXCR5 in CD4 T cells from 2 chronic SIV-infected LNs (left panel). Histograms depicting the expression of CCR7 in bulk CM (solid gray), BCL-6 hiPD-1 $1^{\text {hi }}$, and BCL-6hi CXCR5 dim/hi CD4 T cells (right panel). The frequency of the gated populations is shown. (B) The gating scheme used for identification of particular CD4 populations in LN preparation is shown. The naive and CM cells were analyzed with respect to ICOS and CD150 expression. The naive population was used as reference for setting the gates. The frequency of the gated populations is shown. (C) Confocal images were collected from LN and SP tissues from SIV-negative $(n=2)$ RMs. The CD4, CD20, PD-1, and Ki67 markers were simultaneously analyzed, and their relative localization in 1 SIV-negative $\mathrm{LN}$ is shown (left panel). The boxed area is shown at higher magnification in the top right and the bottom images (scale bars: $100 \mu \mathrm{m})$. The relative distance of CD4 T cells, expressing different amounts of PD-1, from the "center" of the B cell follicle was calculated, and pooled data obtained from 2 SIV-negative SPs are shown (right panel). Each dot represents a cell or a definable part of a CD4 T cell. Bars depict mean $\pm 95 \%$ confidence intervals. 
phenotype inconsistent with GC localization (6). Further analysis of the scatter characteristics and B cell markers on these CXCR5 ${ }^{\text {hi }}$ CD4 T cells calls into question their actual nature (Supplemental Figure 1; supplemental material available online with this article; doi:10.1172/JCI63039DS1). We therefore concentrated on the PD- $1^{\text {hi }}$ CCR $7{ }^{\text {lo }}$ population of CD4 T cells, in which CXCR5 was not detectable, to further characterize RM TFH cells by polychromatic flow cytometry, applying other markers previously used to characterize human and mouse TFH cells (refs. 6, 15, 16, 18 and Figure $1 \mathrm{~B}$ ). The expression of particular receptors on the $\mathrm{CD} 28^{\text {dim }} \mathrm{CD}^{2} 5^{\text {lo }}$ (hereafter referred to as naive) $\mathrm{CD} 4 \mathrm{~T}$ cells was used as a reference to set gates for the identification of particular populations expressing these receptors within the CD28hiCD95 $5^{\text {hi }}$ (hereafter referred to as CM) $(25,26)$ and CD28 ${ }^{\text {lo }}$ CD $95^{\text {hi }}$ memory compartments. Expression of ICOS was highest within the $\mathrm{CD} 28^{\text {hi }} \mathrm{CD} 95^{\text {hi }} \mathrm{CCR} 7{ }^{\text {lo }} \mathrm{PD}-1^{\text {hi }}$ population of CD $4 \mathrm{~T}$ cells, which also showed lower expression of CD150 than other CM CD4 T cell populations (Figure 1B). Most of the CCR7loPD-1 hiICOShi CM CD4 T cells had high levels of CTLA-4 and CXCR4 expression, irrespective of CD150 expression (data not shown). High expression of BTLA and CD69 and diminished expression of CD127 was common in PD- $1^{\text {hi }}$ CM CD4 T cells (Supplemental Figure 2A). Increased mobilization of CD154 was observed in PD- $1^{\text {hi }}$ compared with that in naive and $\mathrm{PD}-1^{\mathrm{lo} / \mathrm{dim}}$ populations after stimulation with SEB (Supplemental Figure 2B).

We next confirmed that PD- $1^{\text {hi }}$ CD $4 \mathrm{~T}$ cells were localized in GCs. Tissues from RMs were analyzed by confocal microscopy for simultaneous expression of CD4, CD20, PD-1, and Ki67. B cell follicle areas surrounded by CD4 T cells were observed in all SP and LN tissues tested. Within these areas, a distinct distribution of PD- $1^{\text {hi }}$ CD4 T cells and Ki67hi cells was observed (Figure 1C and Supplemental Figure $3 \mathrm{~A})$. The majority of Ki67hi cells colocalized with CD20 (Figure 1C and Supplemental Figure 3A), consistent with previously published data in mice (27). Spatial analysis revealed that PD-1 ${ }^{\text {hi }}$ CD 4 cells were localized closer to the follicle center than PD-1 $1^{\text {lo }} \mathrm{CD} 4$ cells, further supporting their definition as TFH cells (Figure 1C). Together, our data show a shared phenotype, at least based on the expression of BCL-6 and the markers we have used, between nonhuman primate and human CD4 TFH cells. Throughout the remainder of the article, we have defined RM TFH cells as CCR7 ${ }^{\text {lo }} \mathrm{PD}-1^{\text {hi }}$ ICOS ${ }^{\text {hi }} \mathrm{CD} 28^{\text {hi }} \mathrm{CD} 95^{\text {hi }} \mathrm{CD} 4 \mathrm{~T}$ cells within the LN and SP. These TFH cells are further subdivided based upon CD150 expression in some experiments.

The TFH cell compartment includes populations with distinct maturation levels and cytokine functions and provides in vitro B cell help. Quantitative PCR revealed increased mRNA expression for MAF $(28,29)$, BCL6 (Figure 2A), and CXCL13 mRNA (Supplemental Figure $3 B$ ) in sorted TFH cells, while TBX21, the major positive regulator of Th1 cells, was found to be downregulated specifically in the CD150 lo $\mathrm{TFH}$ population (Figure 2A). Secreted cytokines from stimulated sorted cells were measured by Luminex assay. PD-1 $1^{\mathrm{lo} / \mathrm{dim}}$ cells were potent producers of Th1 cytokines such as IFN- $\gamma$, TNF, and IL-2 compared with PD- $1^{\text {hi }}$ cells, while both groups secreted MIP-1 $\alpha$ (Figure 2 B). IL-17 was predominantly produced by PD-1 ${ }^{10 / d i m}$ cells (Figure $2 \mathrm{~B}$ ). IL-4 was secreted mainly by CD $150^{\text {lo }} \mathrm{TFH}$ cells, while IL-10 was produced by CD $150^{\text {hi }}$ TFH cells (Figure $2 \mathrm{~B}$ ). We were not able to detect IL-21 production by either flow cytometry or Luminex; however, analysis of IL21 mRNA revealed an increased expression of this transcript in the TFH cell population (Figure 2A). Non-TFH cells were the main source of IL-5 and IL-6 (Supplemental Figure $3 \mathrm{C})$. Coculture of sorted TFH (CCR7 $\left.{ }^{\text {lo }} \mathrm{PD}-1^{\text {hi }}\right)$ or non-TFH (PD-1/o/dim) CD4 T cell populations with autologous LN B cells revealed that TFH cells were able to provide the highest in vitro B cell help, in agreement with previous studies in humans (ref. 30 and Figure 2C). Thus, TFH cells, especially the CD $150^{\text {lo }}$ ones, exhibit a distinct cytokine profile consistent with what has been described in mouse and human studies (4).

Molecular gene signature of TFH cells. Gene expression analysis was performed on RNA from sorted CD150 ${ }^{\text {lo }} \mathrm{TFH}$ and (non-TFH) CCR7 $7^{\text {hi } / l o} \mathrm{PD}-1^{\mathrm{dim} / \mathrm{lo}} \mathrm{ICOS}{ }^{\text {hi }} \mathrm{CD} 150^{\text {lo }} \mathrm{CD} 28^{\text {hi }} \mathrm{CD} 95^{\text {hi }}$ memory CD 4 T cell populations from RM LNs (Supplemental Figure 4A). A 2-way heat map cluster analysis from these populations is shown in Figure 3A and demonstrates that the gene expression signature of CD $150^{\text {lo }} \mathrm{TFH}$ cells is notably different from that of nonTFH cells in LNs. At least 444 genes were differentially regulated between the 2 subsets (http://www.ncbi.nlm.nih.gov/geo/query/ acc.cgi? acc $=$ GSE38795). Significant increases in the expression of BCL6, CXCR5, CTLA4, BTLA, MAF, and CD200 and significant decreases in the expression of $C C R 7, I L 7 R$, and $I L 4 R$ were found (Figure 3A). The gene expression of BCL6, CTLA4, BTLA, CCR7, and $I L 7 R$ is in line with the protein expression of these molecules (Figure 1A and Supplemental Figure 2A), further confirming the gene array data. CD $150^{\text {lo }} \mathrm{TFH}$ cells isolated from uninfected and SIV-infected RMs were transcriptionally distinct, with at least 554 genes being differentially regulated (Figure 3B, left; http:// www.ncbi.nlm.nih.gov/geo/query/acc.cgi?acc=GSE38795). Increased expression of IFN-induced genes (IFI27, IFI44, IFI6, and MX1) and TGF- $\beta$-associated genes (TGIF1 and TGIF2) was observed in CD150 ${ }^{\text {lo }} \mathrm{TFH}$ cells derived from SIV-infected RMs. In contrast, TNFSF14 and FLT3LG, a ligand for FLT3 that mediates maturation of dendritic cells (31), were decreased (Figure 3B). Network analysis of the differentially expressed genes identified a network of interactions associated with IL-4 signaling in CD150 ${ }^{\text {lo }}$ TFH cells that are affected by SIV infection (Supplemental Figure 4B). Several networks (http://www.ncbi.nlm.nih. gov/geo/query/acc.cgi?acc=GSE38795) and biological functions (Supplemental Figure 4C) were differentially regulated in CD $150^{\text {lo }}$ TFH cells from SIV-infected RMs compared with those from noninfected RMs. Overall these data demonstrate unique gene signatures of TFH cells in RMs and show that TFH cells are transcriptionally affected by SIV infection.

SIV infection leads to accumulation of CD4 TFH cells in secondary lymphoid tissues. We next investigated whether SIV infection directly affects the frequency of TFH cells. While the TFH population was unaffected by chronic SIV infection in some RMs (dark gray circles, Figure 4A), the percentage of TFH cells was actually increased in LN tissues from many chronically infected RMs $(29.13 \% \pm 12.89 \%, n=9)$ (red circles, Figure 4A) when compared with that of acute SIVinfected RMs $(7.05 \% \pm 6.79 \%, n=11)$ (light gray circles, Figure $4 \mathrm{~A}$ ) or uninfected RMs $(4.12 \% \pm 1.85 \%, n=10)$ (white circles, Figure $4 \mathrm{~A}$ ). These RMs tended to maintain their frequency of total CD4 (Figure 4C) and CM (Figure 4C) T cells at the expense of naive (Figure 4C) and CCR7hiPD-1 ${ }^{\text {lo }}$ (non-TFH) CD4 T cells (Figure 4A). Similar data were obtained from SP tissues (data not shown). We found that half the chronically infected RMs had TFH levels consistent with those of uninfected RMs, while the other half had elevated TFH levels. We therefore divided the SIV-infected RMs into 2 groups of equal size (one having high levels of TFH cells and the other having low levels of TFH cells; referred to herein as high and low TFH, respectively) 
A
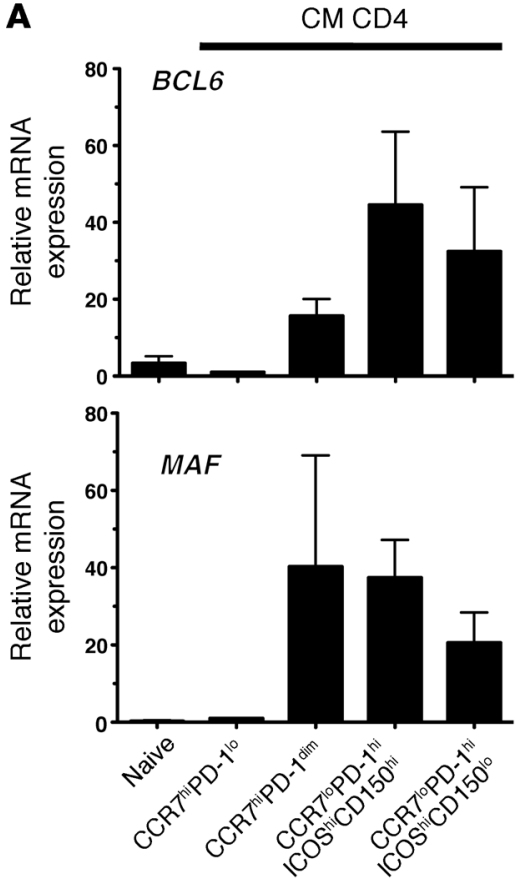

B
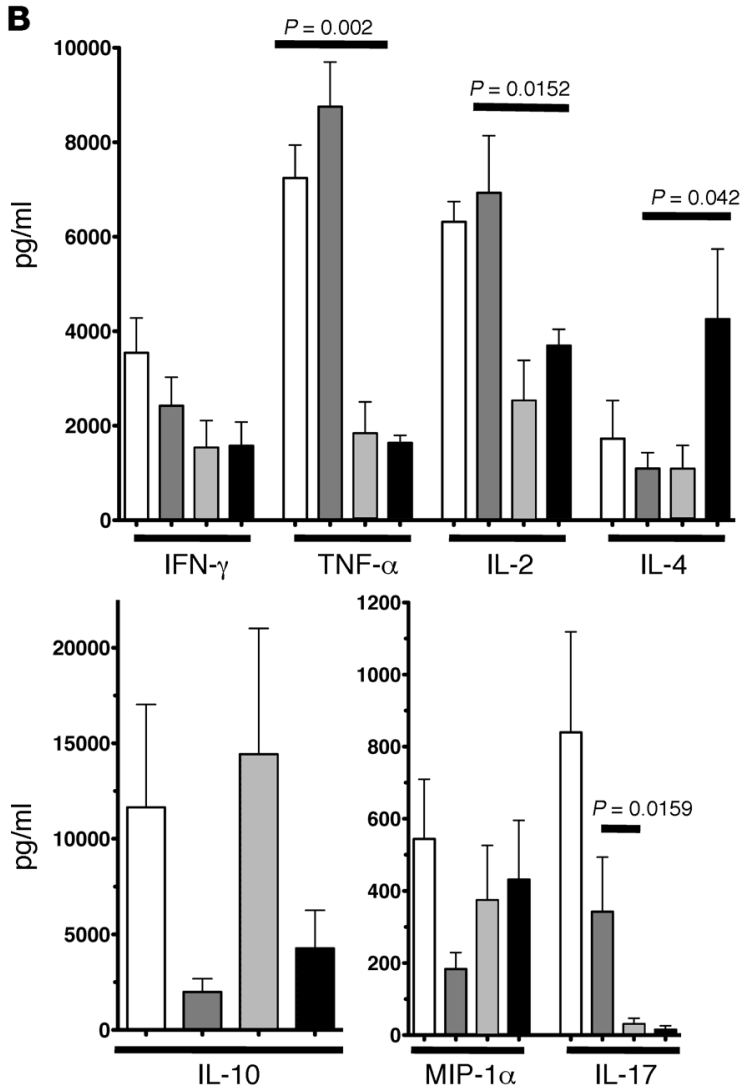

IL-10

IL-17
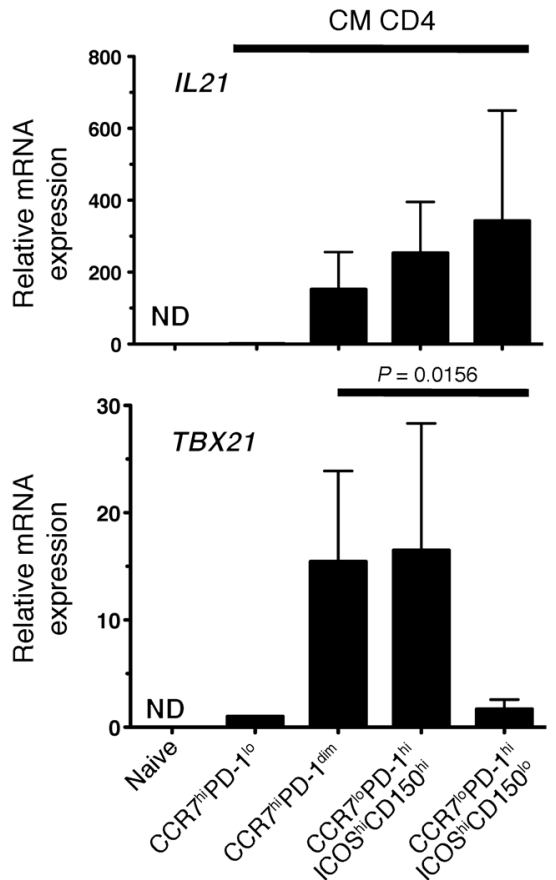

CCR7 hildimPD-1/d/dim ICOShiCD150

$\square$ CCR $7^{\text {hidimPD- }} 1^{\text {lo/dim } / C O S}{ }^{\text {hiCD } 150^{10}}$

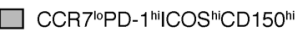

CCR7'opD-1 $1^{\mathrm{hi}} \mathrm{COS}^{\mathrm{ni}} \mathrm{CD} 150^{10}$

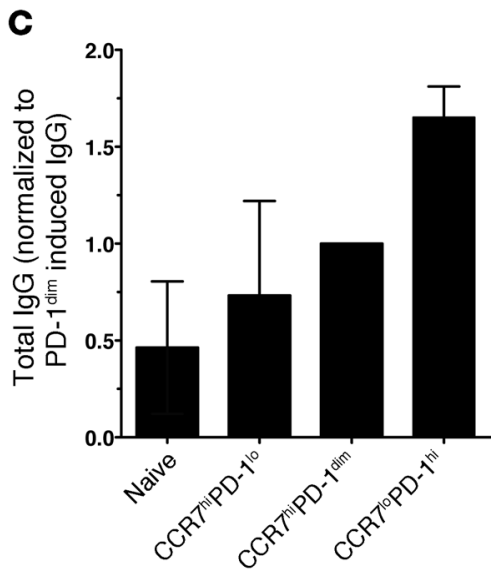

tion of TFH cells during chronic SIV infection occurred predominantly within the CD $150^{\text {lo }} \mathrm{com}$ partment (Figure 4D). This accumulation of TFH cells in chronic SIV infection was accompanied by preserved architecture and distribution of TFH and B cells in lymphoid follicles (Supplemental Figure 4D). We found no expansion of $\mathrm{T}$ follicular regulatory cells (32) during SIV infection, as FOXP3 was found to be expressed in only a minor population $(5.9 \% \pm 4.06 \%)$ of sorted CD150hi TFH cells, and this population was even smaller $(1.3 \% \pm$ $1.57 \%$ ) when the coexpression of FOXP3 and CD25 was analyzed (Supplemental Figure 5A). In addition there was no correlation

(Figure 4A). BCL-6 expression was found to be significantly increased in TFH cells from the high group compared with that in those from the low TFH group (Figure 4B). Further defining the TFH cells based upon CD150 expression demonstrated that the accumula- between plasma viral load and the relative frequency of TFH cells (data not shown). Our data indicate that TFH cells are preserved or increased compared with other CD4 T cell subsets within secondary lymphoid tissues during chronic SIV infection. 
A

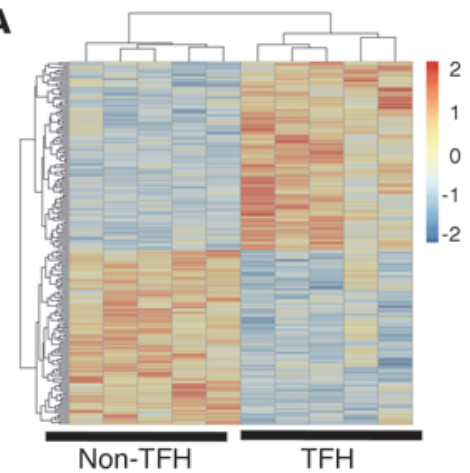

B

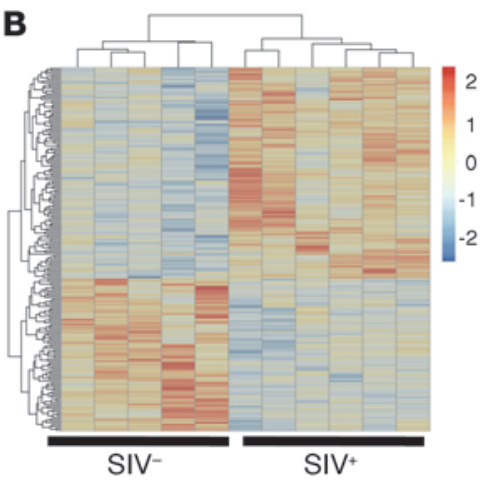

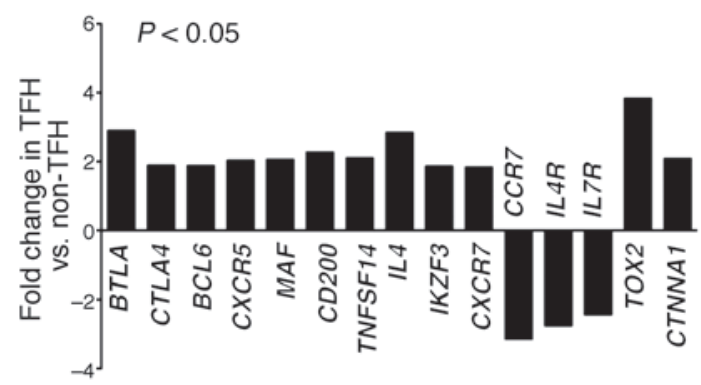

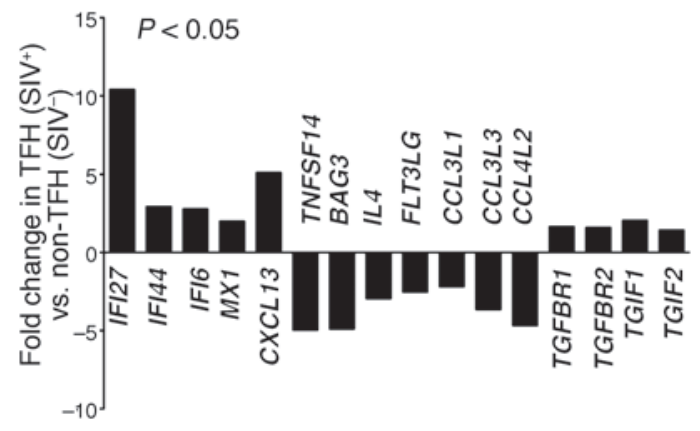

Figure 3

RM TFH cells exhibit a distinct transcriptional profile compared with that of non-TFH cells. (A) Heat map clustering analysis of gene expression in non-TFH $(n=5)$ compared with TFH $(n=5)$ cell populations from SIV-negative $(n=5)$ tissues. The top 200 genes, based on raw $P$ value for the contrast, are shown. The relative expression (fold change) of selected genes $(n=15)$ between TFH and non-TFH populations is shown. Genes were significantly differentiated with fold change of more or less than 1.5 and raw $P$ value of less than 0.05 . (B) Heat map analysis of gene expression between TFH cells from SIV-negative $(n=5)$ and chronic SIV-infected $(n=6)$ tissues. The relative expression of selected genes $(n=15)$ is shown.
CD4 TFH cells are susceptible to SIV infection. We next determined whether the preservation of TFH cells during SIV infection was a result of low in vivo infectivity. Multiple CD4 T cell populations were sorted from RM mesenteric LNs during acute and chronic SIV infection. Higher numbers of SIV-gag DNA copies were found in $\mathrm{CD} 150^{\text {hi }} \mathrm{TFH}$ cells compared with those in CD150 $0^{\text {lo }} \mathrm{TFH}$ $(P=0.0078)$ or CCR7hi/lopD-1 ${ }^{\operatorname{dim}}(P=0.0156)$ CD4 T cells during acute SIV infection (Figure 4E). These differences in the in vivo infection levels did not persist into chronic SIV infection (Figure 4E). Our results indicate that TFH cells are as susceptible to SIV as are non-TFH cells within RM LNs, yet they are often maintained or increased in frequency and spatial localization within B cell follicles well into chronic infection.

CD150 ${ }^{l o}$ TFH cells are characterized by low in vivo cycling and increased sensitivity to in vitro cell death. We next investigated potential cellular factors that could regulate the accumulation/preservation of TFH cells in chronic SIV infection. First, the ability of TFH cells to progress through the cell cycle was investigated by measuring the in vivo incorporation of BrdU in CD4 $\mathrm{T}$ cell populations from LNs of chronically SIV-infected RMs (Figure 5A). All CD4 T cell populations were relatively stable during the 3 -week experiment (data not shown). Naive CD4 T cells showed low BrdU incorporation $(0.445 \% \pm 0.21 \%$ at day 3$)$ compared with that of the CD28 2 hi CD95 ${ }^{\text {hi }}(11.72 \% \pm 1.3 \%$ at day 3$)$ and $C D 28^{\text {lo }}$ CD $95^{\text {hi }}(10.99 \% \pm 2.91 \%$ at day 3) populations (Figure 5A). Interestingly, CD $150^{\text {lo }} \mathrm{TFH}$ cells showed low uptake of $\operatorname{BrdU}(5.93 \% \pm 1.23 \%$ at day 3$)$ even compared with CD 150 hi $\mathrm{TFH}$ cells $(25.9 \% \pm 5.27 \%$ at day 3$)$ (Figure $5 \mathrm{~A})$. Next, spontaneous expression of active caspase- 3 was used to investigate the in vitro survival of these cellular populations. As expected, the naive population showed the lowest spontaneous caspase- 3 activity (Figure 5B). The CD150lo TFH cells exhibited the highest spontaneous caspase- 3 activity $(20.31 \% \pm 5.49 \%)$, even compared with that of the CD $150^{\text {hi }}$ TFH $(3.81 \% \pm 0.88 \%)$ cells, and SIV infection did not affect this finding (Figure 5B). A major regulator of TFH survival is the interaction between PD-1 with its ligand, PD-L1, on B cells (33). We found that chronic SIV infection was accompanied by significantly reduced frequency of PD-L $1{ }^{\text {hi }} \mathrm{B}$ cells in naive and memory $\mathrm{B}$ cell compartment in RM LNs (Figure 5C and Supplemental Figure $5 \mathrm{~B})$. We also investigated the possible role of altered trafficking in the accumulation of TFH cells during SIV infection by assessing the mRNA expression of S1P receptor 1 (S1PR1) in sorted cells (34). TFH cells, specifically those with low CD150 expression, were characterized by very low expression of S1PR1, both in acute (Figure 5D) and chronic SIV infection (Figure 5D). However, a marked induction of S1PR1 was found on non-TFH cells from SIV-infected RMs with a high percentage of TFH cells (Figure 5D). When cells from noninfected RMs were analyzed, the highest expression of S1PR1 was found in naive cells (Supplemental Figure 5C). Thus, CD150 hi TFH cells have high turnover and low spontaneous cell death, while CD150 lo TFH cells have low turnover and high spontaneous cell death. Both populations appear to have a reduced ability for recirculation into the periphery.

Skewed IL-6-induced signaling in the presence of high immune activation is associated with accumulation of TFH cells in chronic SIV infection. We next assessed whether the general immune activation associated with SIV infection (35) could be operative in driving the observed accumulation of TFH. The level of plasma soluble CD14 (sCD14), a marker of immune activation (36), was significantly higher in the group of SIV-infected RMs in which TFH cells had accumulated (Figure 6A). Notably, these levels of sCD14 were comparable to the levels during acute SIV infection (Figure 6A). IL-6, a proinflammatory cytokine that is a critical regulator of TFH cells $(19,21,22,37)$, is increased in HIV infection (38). In line with the sCD14 levels, we found significantly increased levels of plasma IL- 6 in chronically 


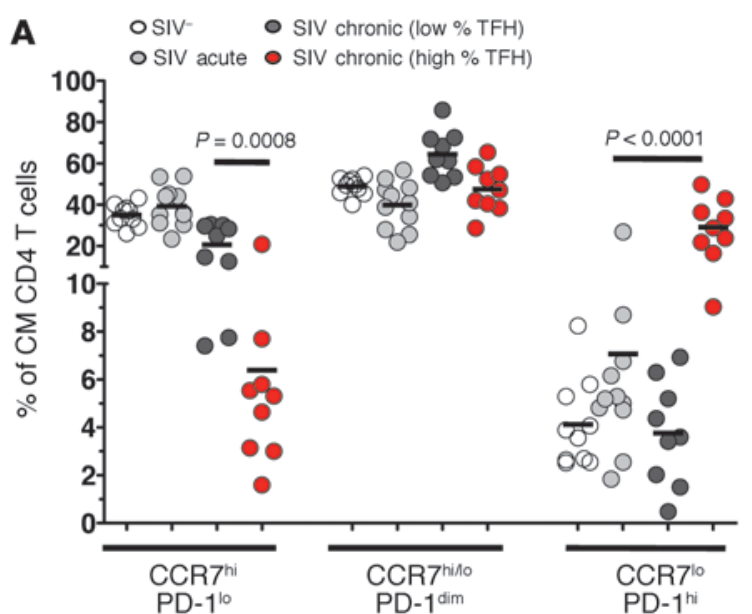

C

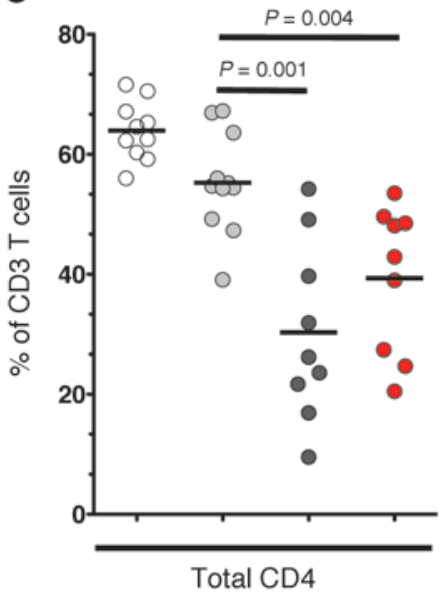

$\mathbf{E}$

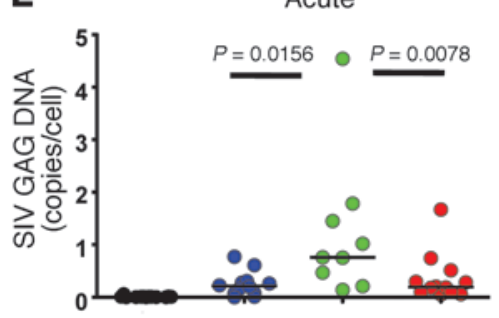

B

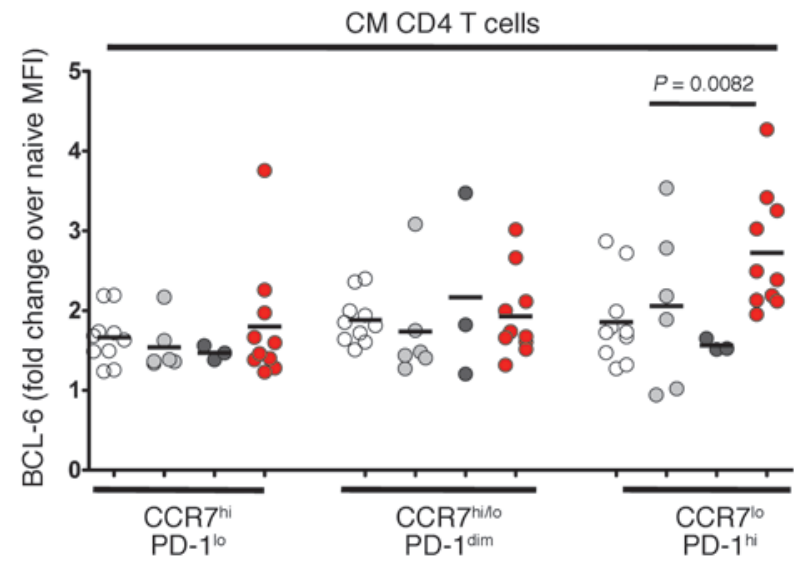

D

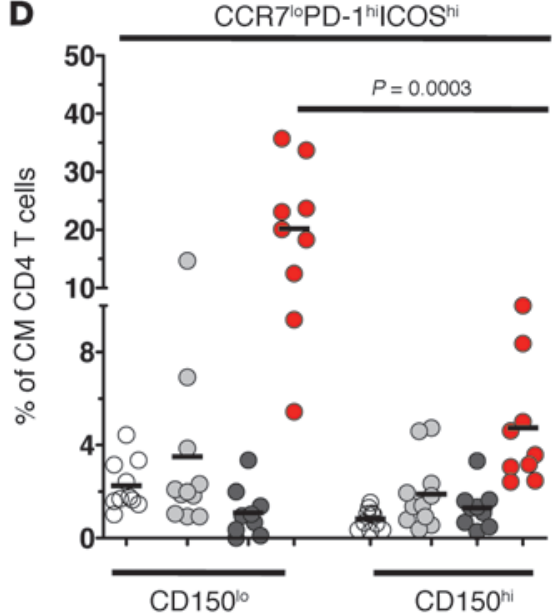

Figure 4

Accumulation of TFH cells during chronic SIV infection. (A) Pooled data showing the frequency (\%) of CCR7 hiPD- $1^{\text {lo }}$, CCR7 $7^{\text {hilloPD- }} 1^{\text {dim }}$, and CCR7 ${ }^{\text {loPD }} 1^{\text {hi }}$ populations in the CM cellular compartment from LN tissues from noninfected (white; $n=10$ ), acute SIV-infected (light gray; $n=11$ ), and chronic SIV-infected RMs (dark gray: low percentage of TFH cells, $n=9$; red: high percentage of TFH cells, $n=9$ ). (B) Pooled data showing the levels (MFI) of BCL-6, expressed as fold change over the MFI of naive cells, in CD4 T cell subsets. (C) Pooled data showing the frequency (\%) of total, naive, and CM CD4 T cells. (D) The frequency (\%) of the CD150 lo and CD150hi TFH cells is shown. (E) SIV-GAG DNA was measured by PCR. LN tissues from acute SIV-infected (samples collected on day 3, 7, 10, 14, 17, and 21 after infection) ( $n=12$ ) and chronic SIVinfected $(n=6)$ RMs were used. Each dot represents data for a single LN from an individual animal. Animals are represented only once in each plot. Horizontal lines depict either $(\mathbf{A}-\mathbf{D})$ mean or $(\mathbf{E})$ median values. $P$ values were calculated using the $(\mathbf{A}-\mathbf{D})$ Mann-Whitney $U$ test or the $(\mathbf{E})$ Wilcoxon matched-pairs signed-rank test.

SIV-infected RMs compared with those in noninfected animals (Figure 6B). There was no difference in the IL-6 levels between the high and low TFH groups of chronically infected animals (data not shown). However, the expression of IL-6Ra on TFH cells was increased in SIV-infected RMs with high percentage of TFH cells
(Figure 6C and Supplemental Figure 5D). In fact, the expression of IL-6Ra and the relative frequency of TFH were directly correlated (Figure 6D). In addition, the catalytic IL-6R subunit (GP130) was specifically upregulated on TFH cells (Supplemental Figure 5E), and we found high levels of mRNA encoding cytosolic regulators 
of the IL-6R subunits (data not shown), both of which support the expression profile of the IL-6R complex in TFH. Although with different kinetics (39), IL-6 induces phosphorylation of both STAT- 1 and STAT- 3 in T cells (40). Therefore, we investigated the phosphorylation of these 2 factors in CD4 T cell populations from RM LNs after in vitro treatment with IL-6. Phosphorylation of both STAT-1 and STAT-3 was found after IL-6 treatment (Figure 6E and Supplemental Figure 6, A and B). A significantly higher phosphorylation of STATs was observed in chronically SIV-infected RMs compared with that in uninfected and acutely infected RMs (Figure 6E). Although activation of both STATs was found in chronic SIV infection, the phosphorylation of STAT-1 and the ratio of phosphorylation of the 2 molecules (pSTAT-1/pSTAT-3) in the PD-1hi compartment were both inversely correlated with the frequency of TFH cells (Figure 6F, top and bottom panel, respectively). A similar profile of STAT phosphorylation was found when cells were treated with IL-21, an inducer of STAT-1 and STAT-3 (ref. 41 and data not shown).

While IL-6 did not affect the survival of sorted CD4 T cells stimulated in vitro with an anti-CD3 antibody, the survival of TFH cells was dramatically reduced under these conditions compared with that of the other CD4 T cell populations (Figure 6G, left). However, IL- 6 was able to boost the absolute numbers of both naive and non-TFH CD4 T cells after anti-CD3 stimulation (Figure $6 \mathrm{G}$, right). In line with their compromised in vivo cycling, this increase in absolute numbers was not seen in the TFH compartment whether or not IL-6 was added (Figure 6G, right). In support of the proliferative effect of IL-6, increased expression of Ki67 and in vitro incorporation of BrdU by naive and non-TFH CD4 T cells was found in the presence of IL-6 (Supplemental Figure 6C). Next, the possible effect of IL- 6 on TFH-related molecules was investigated. In vitro treatment with IL- 6 in the presence of anti-CD3 accelerated the activation of CD4 $\mathrm{T}$ cells, as judged by a reduction in the $\mathrm{CD} 5^{\text {lo }}$ population (Supplemental Figure 7A). Furthermore, IL-6 induced the expression of BCL-6 in both CD95 ${ }^{\text {lo }}$ and CD95 ${ }^{\text {hi }} \mathrm{CD} 4 \mathrm{~T}$ cell populations, especially after 72 hours of treatment (Figure 6H). IL-6 further upregulated the anti-CD3-induced expression of ICOS, CD95, and PD-1, especially in the CD95 ${ }^{\text {hi }}$ CD4 $\mathrm{T}$ cells (Supplemental Figure 7B). Collectively, these data are consistent with increased IL- 6 signaling under SIV-associated immune activation driving cells toward a TFH phenotype.

Accumulation of TFH cells is associated with increased frequency of $G C$ $B$ cells and SIV-specific immunoglobulins. Given the critical role of TFH cells in regulation of GC B cell responses (1), we sought to investigate the phenotype of B cells in RM LNs. Increased frequency of total B cells was found in chronic SIV infection, especially in the animals with high TFH (Supplemental Figure 8A, left). Accumulation of TFH was associated with a lower frequency of naive (CD27lo IgDhi) B cells (Supplemental Figure 8A, middle), preserved surface expression of IgG (Supplemental Figure 8A, right), and significantly increased activation of B cells, as judged by Ki67 expression (Supplemental Figure 8B). We assessed GC B cells by binding of peanut agglutinin (PNA) (42). As expected, no PNA staining was observed on B cells in PBMCs (Figure 7A, left). Accumulation of TFH cells was associated with a significant increase in PNA ${ }^{\text {hi }} \mathrm{B}$ cells, especially those not expressing surface IgG (Figure 7A, right). Furthermore, activated (Ki67 hi) B cells expressed increased levels of BCL-6 (Figure 7B). In addition, the frequency of BCL- $6^{\text {hi }} \mathrm{B}$ cells was significantly increased in chronically SIV-infected RMs, which had accumulated TFH cells (Figure 7B, right). We then analyzed the SIV-specific immunoglobulins in the plasma from acute and chronically SIV-infected RMs. Accumulation of TFH cells was associated with significantly higher titers of both IgG and IgA antibodies against several SIV antigens (Figure 7C). Furthermore, SIVspecific IgGs were characterized by higher avidity in RMs with high TFH (Figure 7D and Supplemental Figure 8C). Taken together, our data demonstrate that accumulation of TFH cells in chronic SIV infection is accompanied by dramatic expansion of GC B cells and increased titers of higher avidity SIV-specific immunoglobulins.

\section{Discussion}

We have identified a CD4 T cell population in RM secondary lymphoid tissues that resides in the $\mathrm{B}$ cell follicle and is characterized by a CD28 ${ }^{\text {hi }} \mathrm{CD} 95^{\mathrm{hi}} \mathrm{CCR} 7^{\mathrm{lo}} \mathrm{PD}-1^{\text {hi }}$ phenotype. Increased levels of ICOS, CTLA-4, CXCR4, BTLA, CD69, CD154, and BCL-6, as compared with the bulk of CD4 $\mathrm{T}$ cells, were typical of this population, which is similar to human and mouse TFH cells $(6,18,43)$. We have further identified GC structures in LN and SP, which are surrounded by $\mathrm{CD} 4 \mathrm{~T}$ cells possessing a PD- 1 hi phenotype. The relative distribution of PD-1, CD20, and Ki67 within these structures is indicative of GC "dark" and "light" zones, in which somatic mutation and affinity maturation of the $\mathrm{B}$ cell response is facilitated by TFH cells (2). Although TFH cells defined by the multiple markers that we used expressed high CXCR5 mRNA levels, the use of CXCR5 to define TFH cells proved problematic (Figure 1A and Supplemental Figure 1). Despite this difficulty, the panel of markers that we used clearly identifies a population of CD4 T cells with a phenotype, function, and location indicative of TFH cells.

CD150 is an important regulator of TFH development, through its interaction with the adaptor SAP $(14,44)$. Reduced TBX21 transcription in CD150 ${ }^{\text {lo }} \mathrm{TFH}$ cells was associated with high production of IL-4, while TFH cells with high expression of CD150 predominantly produced IL-10. In addition, TFH cells produced significantly fewer Th1 cytokines compared with non-TFH cells, irrespective of CD150 expression (Figure 2B). Our data are therefore consistent with CCR7 ${ }^{\text {lo }} \mathrm{PD}-1^{\text {hi }}$ ICOS $^{\text {hi }} \mathrm{CD} 150^{\text {lo }} \mathrm{CD} 4 \mathrm{~T}$ cells being the "GC TFH" cells in RMs (45). Because IL-4 is induced upon CD150/SAP crosstalk and is mediated by FYN and PKC- $\theta$ / NF- $\kappa B$ signaling $(13,46)$, investigating the ratio of CD150 to SAP and the relative function of FYN- and PKC- $\theta$-mediated pathways could inform the mechanisms underlying these polarized cytokine profiles. We found that sorted CCR ${ }^{\text {lo }} \mathrm{PD}-1^{\text {hi }} \mathrm{CD} 4 \mathrm{~T}$ cells from chronically infected RMs were capable of providing in vitro help to sorted total or memory autologous B cells, further supporting their definition as TFH cells. Further experiments, using a larger cohort of noninfected and SIV-infected RMs, are needed to understand the nature of the in vitro help (30) and compare it to the help provided by TFH cells from noninfected RMs.

TFH cells from SIV-infected and uninfected RMs were transcriptionally different. The increased expression of the IFN-induced genes in TFH cells from SIV-infected RMs suggests that these cells respond to the increased IFN production associated with the SIV microenvironment. In addition, the increased expression of TGF- $\beta$-associated genes suggests a role of TGF- $\beta$ as a regulator of TFH dysfunction in SIV-infected RMs (47). Other indicators of TFH dysfunction in SIV-infected RMs include the decreased expression of TNFSF14 and FLT3LG. Finally, we show significant reduction in the expression of the $I L 4$ gene in TFH cells from SIV-infected RMs; this gene plays a critical role in memory B cell survival and isotype switching. Our data therefore provide critical 
A

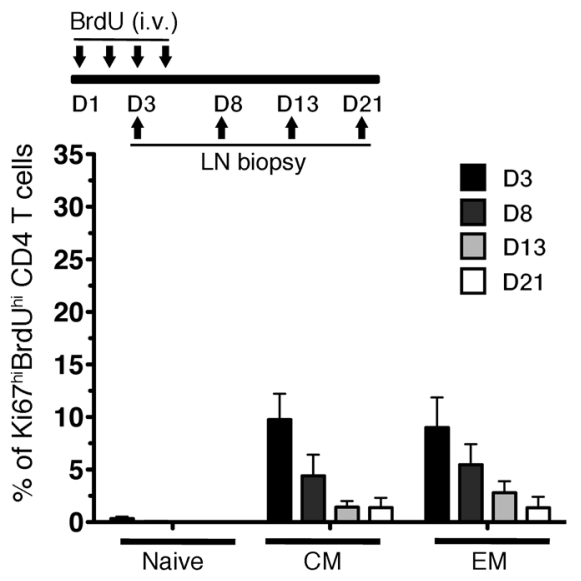

B
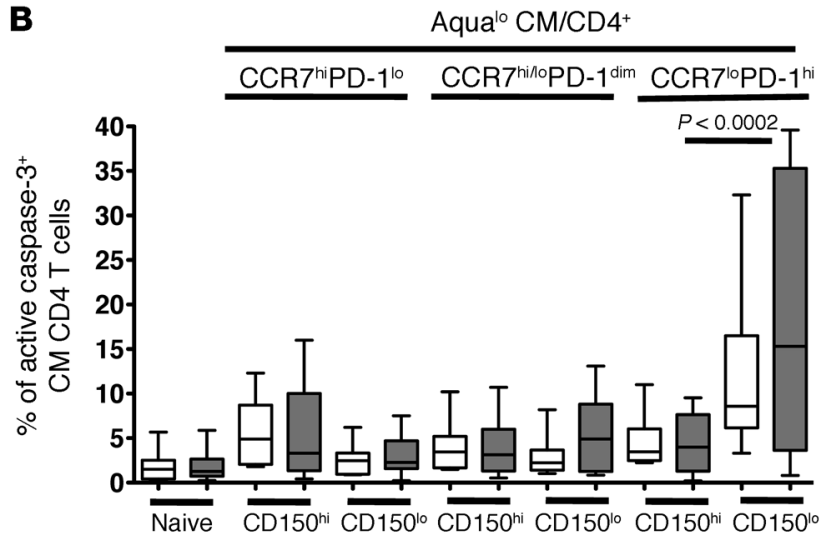

CM CD4 T cells

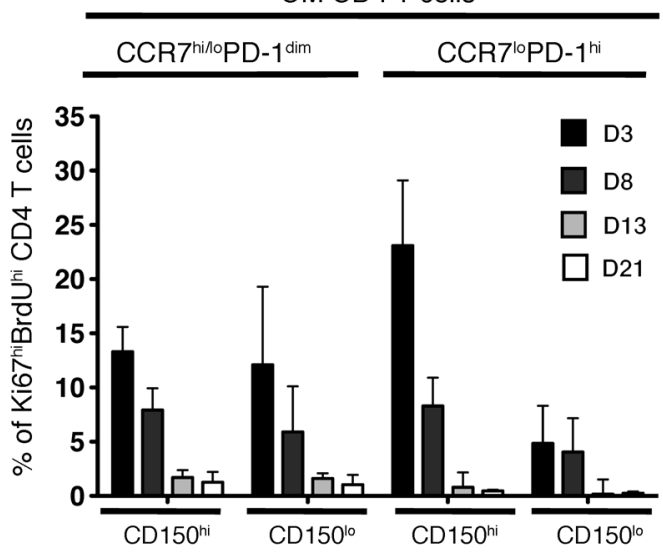

C

D
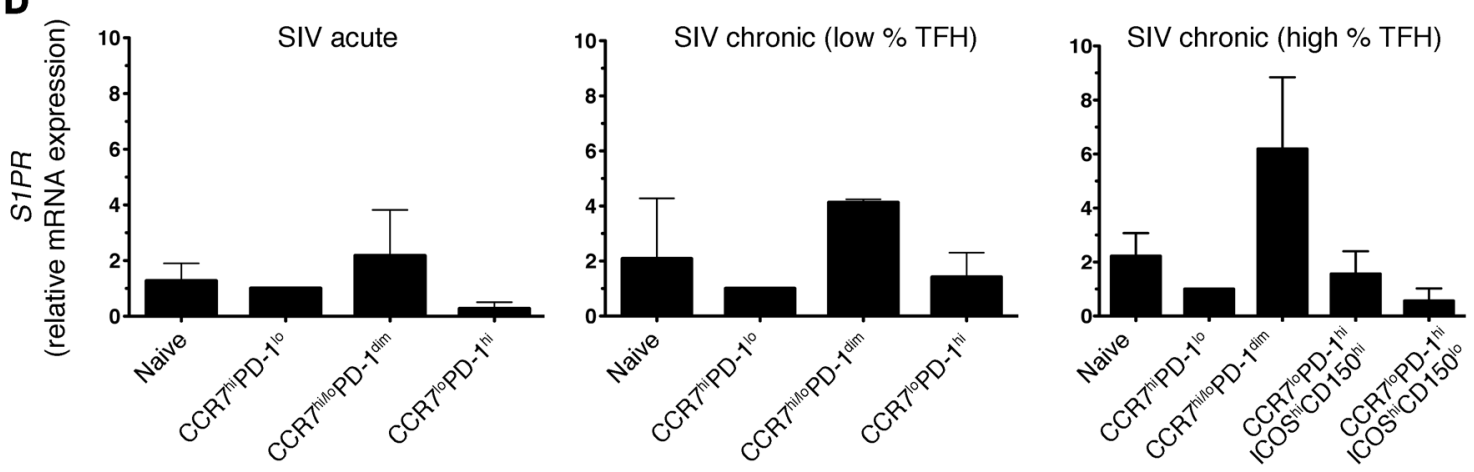

Figure 5

TFH cells are characterized by low cell cycling capacity and increased sensitivity to in vitro cell death. (A) The schedule of the in vivo administration of BrdU and collection of LN tissues from chronic SIV-infected RMs $(n=3)$ as well as the percentage of Ki67 hiBrdUhi cells in naive, CM, and EM CD4 T cell populations are shown. The frequency (\%) of Ki67hiBrdUhi cells in CM/CD4 T cell populations, based on their expression of CCR7, PD-1, and CD150, is also shown. (B) Pooled data showing the percentage of active caspase- $3^{+}$cells in the naive and CM subpopulations from noninfected ( $n=7$; white bars) and chronic SIV-infected RMs ( $n=13$; gray bars). (C) Pooled data showing the relative frequency (\%) of PD-L1 ${ }^{\text {lo }}$, PD-L1 dim, and PD-L1 ${ }^{\text {hi CD20 }}{ }^{\text {hi CD }}{ }^{\text {lo }} \mathrm{B}$ cells in SIV $^{-}(n=10)$ and chronic SIV-infected LNs $(n=10$ for low percentage of TFH cells and $n=4$ for high percentage of TFH cells). (B and $\mathbf{C}$ ) In the box-and-whiskers plot, box size represents the limits of data for the second and third quartiles, with medians shown as bars. Whiskers define the minimum and maximum of the data presented. $P$ values were calculated using the Mann-Whitney $U$ test. ${ }^{*} P<0.0001$. (D) The relative expression of the S1PR1 mRNA in sorted cells from acute SIV-infected $(n=3$; LN tissues) and chronic SIVinfected RMs, with low percentage of TFH cells ( $n=3$; LN tissues) and high percentage of TFH cells ( $n=7$; LN tissues), is shown. The normalized CT value for $C D 28^{\text {hi }}$ CD95 ${ }^{\text {hi }}$ CCR $7{ }^{\text {hiPD }}-1^{10}$ cells was assigned a value of 1 , and the fold change over this value for the populations tested is shown.

information regarding molecular pathways that regulate TFH cells in vivo and demonstrate that many of them are adversely affected by SIV infection, possibly leading to downstream problems with the $\mathrm{B}$ cell response to SIV and other infections.
Analysis of multiple CD4 $\mathrm{T}$ cell populations revealed loss of CCR7 ${ }^{\text {hiPD }} 1^{\text {lo }}$ accompanied by expansion of CCR7 ${ }^{\text {lo }}$ PD- $1^{\text {hi }}$ (TFH) cells in secondary lymphoid tissues during chronic SIV infection. This was more evident within the CD150 lo TFH compartment. 
Our assays only allow a determination of the relative frequency of particular T cell populations. Because the relative accumulation of TFH cells was observed in the presence of total CD4 T cell loss, we cannot determine whether there is an absolute increase, or just less loss, of TFH cells compared with other CD4 T cells in LNs during SIV infection. We expected to find that TFH cells were less infected in vivo than other CD4 T cell populations in the LN. In fact, we found the opposite. During acute SIV infection, LN TFH cells were frequently in cell cycle (expressed Ki67) and had greater copy numbers of SIV than other CD4 T cell populations (Figure $4 \mathrm{E}$ and data not shown). No differences were found, however, during the chronic SIV infection, indicating that infection per se is not a major regulator of the TFH cell expansion. We found that CD150 ${ }^{\text {lo }}$ TFH cells are not self-renewable, while their capacity for egression into the blood stream, judged by the expression of S1PR1, is compromised. Interestingly, the expression of S1PR1 was low in TFH cells regardless the infection status, indicating that this is an intrinsic characteristic of these cells. However, it was apparent that chronic SIV infection results in increased S1PR1 expression on non-TFH CD4 T cells, potentially leading to increased egression of these cells into periphery. We hypothesize that such a process could affect the dynamics of CCR7 $7^{\text {hiPD }}-1^{\text {lo }}$ and CCR7hi/lopD- $1^{\text {dim }}$ CD 4 $\mathrm{T}$ cells in LNs from RMs with high TFH (Figure 4A). Furthermore, the rate of spontaneous cell death of TFH cells, as measured by active caspase- 3 expression, was unaffected by SIV. The high level of spontaneous active caspase- 3 expression in CD150 lo TFH cells suggests that they normally have a very short half-life in vivo, possibly explaining why SIV infection does not impact further upon an already short survival. In fact, our data argue that TFH cells may be less exposed to death signals mediated by PD-L1 expressed on B cells (33) during the chronic SIV infection (Figure 5C).

Within the group of SIV-infected RMs who had a high frequency of TFH cells, we found significantly elevated levels of sCD14, supporting the potential role of general immune activation in this process. In particular, our data point to increased IL-6 signaling that favors the development of TFH. The increased expression of the IL-6R complex found on TFH cells, especially in chronic SIV infection, was associated with increased plasma levels of IL-6. Treatment with IL- 6 significantly increased the proliferation of naive and non-TFH cells but had no effect on their survival. In line with their general lack of in vivo cycling, no proliferation was observed in stimulated TFH cells in the presence or absence of IL-6. Furthermore, IL-6 was capable of inducing molecular markers (BCL-6, ICOS, CD95, PD-1) of TFH cells, especially after TCR stimulation. Our gene analysis revealed that $C E B P A$, a major negative regulator of $\mathrm{G}_{1} / \mathrm{S}$ transition (48-50) and necessary factor for the expression of IL-6Ra $(51,52)$, was noticeably increased in the CD150 ${ }^{\text {lo }}$ TFH cells. Proliferation and differentiation can be mutually exclusive processes, and CEBPA has been proposed to serve as a linker between these two biological pathways (53). Therefore, we hypothesize that CEBPA could serve as a central coordinator of the TFH dynamics in vivo by regulating their proliferation/differentiation process as well as the activity of IL- 6 during the development of TFH responses. IL- 6 signaling is mediated by phosphorylation of both STAT-1, a major inducer of Th1 cells (54), and STAT-3, a critical regulator of TFH $(21,22)$. Although ex vivo IL- 6 treatment induced phosphorylation of both STAT- 1 and STAT- 3 in all populations tested, a significantly higher STAT-3 phosphorylation was found in TFH cells compared with that in non-TFH cells only in samples collected from chronically infected RMs. Furthermore, mobilization of STAT-1 in TFH cells, under IL- 6 treatment, was selectively compromised during chronic SIV infection. Whether increased expression of IL-6Ra or altered intracellular interactions in LNs with high TFH cells are responsible for this skewed IL-6 signaling needs to be investigated. Our in vitro data indicate that IL-6 could affect the development of TFH responses in SIV infection at multiple stages by affecting the molecular program (i.e., BCL-6 expression) and proliferation of less differentiated CD4 T cells as well as the intracellular signaling (through STAT phosphorylation) in TFH cells. Thus, our data reveal altered IL-6 signaling during chronic infection that favors the development of TFH cells and further support the significance of IL- 6 as a critical regulator of TFH cell dynamics.

Accumulation of TFH cells in chronic SIV infection was associated with expansion of the GC B cell compartment and increased circulating SIV-specific immunoglobulins, in agreement with recently described data (24). Although not significant, the avidity of SIV-specific antibodies was higher in RMs with high TFH compared with that in RMs with low TFH. Our data show that the gene expression alterations (i.e., reduction of IL4) imparted by SIV upon the TFH cells did not affect the ability of B cells to respond to SIV. Therefore, delineating the molecular mechanisms mediating the TFH-B cell interactions is critical for our understanding of the development of neutralizing activity against SIV and presumably HIV. Moreover, further work is needed to understand whether the higher infection levels of TFH cells in the acute phase contributes to the limited specificities of the initial HIV-1-specific (and SIV-specific) antibody responses (55).

In summary, we have characterized TFH cells in RMs at the phenotypic, molecular, and tissue localization level. Our findings support a model of TFH cell dynamics in which a constant flow of activated CD4 T cells enter the TFH compartment followed by further differentiation to a status (CD150 $\left.{ }^{\text {lo }} \mathrm{TFH}\right)$ characterized by a polarized cytokine profile and reduced survival ability. SIV impacts upon the molecular gene signature of this cell population yet does not lead to their specific depletion and often results in their accumulation. This is likely because of the diverse populations of CD4 T cells that can serve to continually replenish this population, thereby assuring their persistence late into SIV infection.

\section{Methods}

\section{Animals}

Indian RMs (Macaca mulatta) (Covance Research Products) were handled in accordance with the standards of the American Association for the Accreditation of Laboratory Animal Care. RMs were infected intravenously with a SIVmac251 stock. A previously described $(56,57)$ cohort of acute SIVinfected $(n=13)$ RMs was used for the analysis of CD4 T cell population infection rates during the acute phase.

\section{Antibodies}

We used the following directly conjugated antibodies: CD3-Cy7APC (SP34-2), CD95-Cy5PE (DX-2), PD-L1 Cy7PE (MIH-1), BCL-2-PE (BCL-2/100), CTLA4-APC (BNI3), CD27-Alx700 (catalog no. 560611), CD154-PE (TRAP1), active caspase-3-FITC (catalog no. 51-68654X), BrdU-APC, (catalog no. 51-23619L), Ki67-FITC (catalog no. 556026), Ki67 Alx700 (catalog no. 561277), CD126 (IL-6Ra)-PE (catalog no. 551850), CD130 (GP130)-PE (catalog no. 555757), STAT-1-FITC (pY701) (catalog no. 612596), and STAT-3-Pacific Blue (pY705) (catalog no. 560312) (all from BD Biosciences); CD28-ECD (CD28.2) 


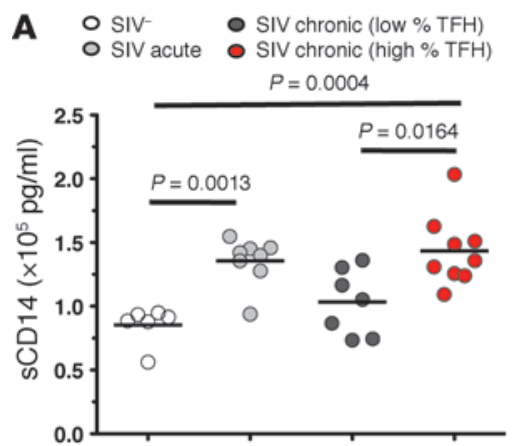

B

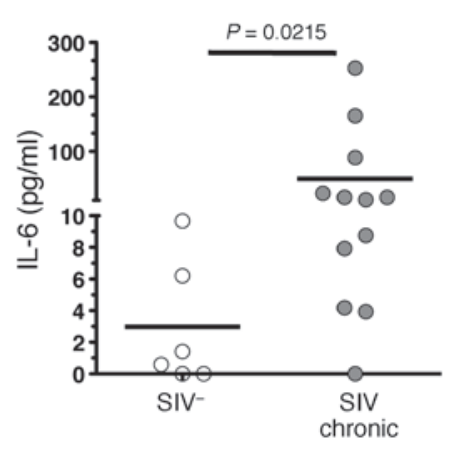

E

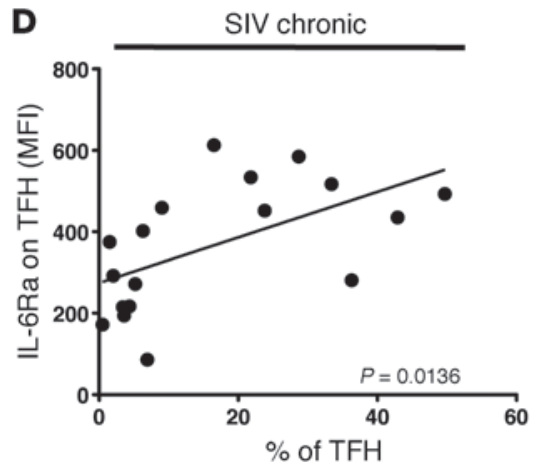

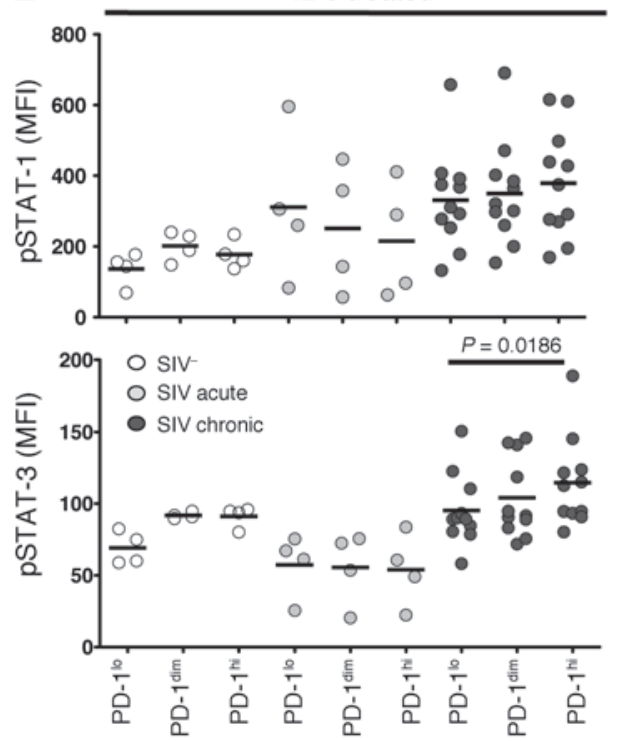

C

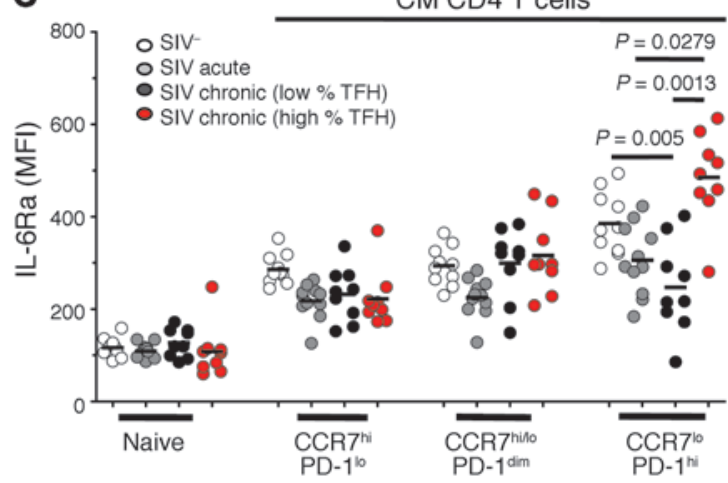

F
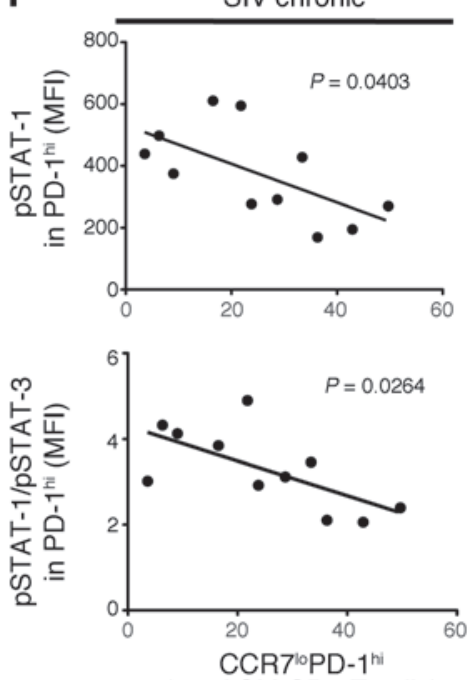

(\% of CM CD4 T cells)
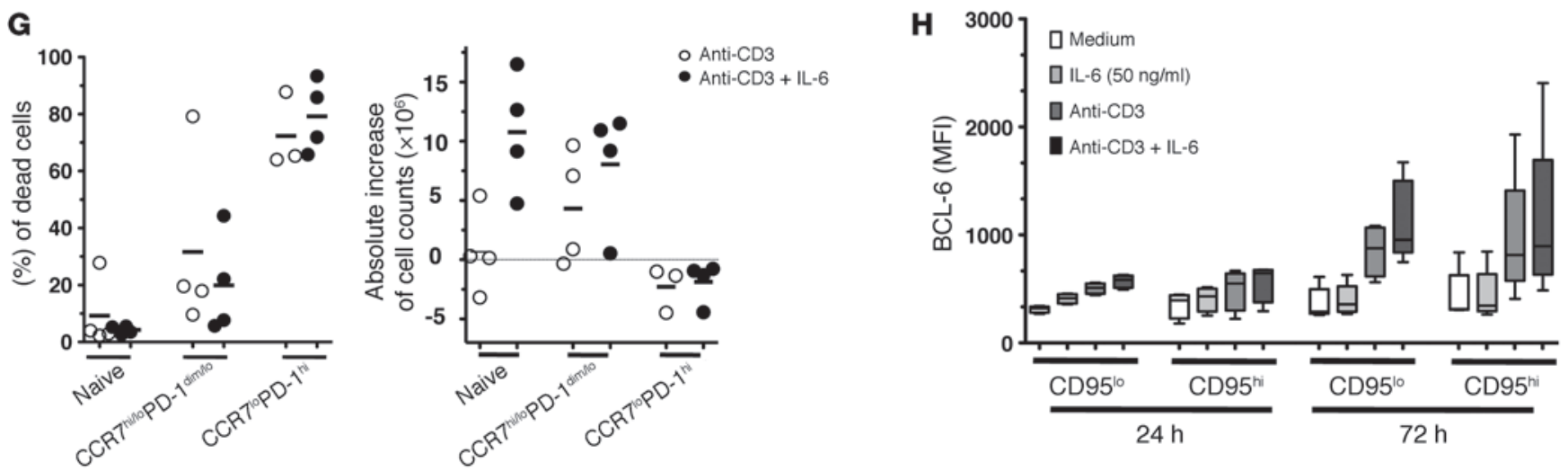

Figure 6

TFH cell accumulation correlates with increased immune activation and skewed IL-6 signaling. (A) Plasma sCD14 levels from noninfected $(n=6)$, acute $(n=8)$, and chronic SIV-infected RMs (dark gray: low TFH cells, $n=7$; red: high TFH cells, $n=9$ ). (B) Plasma IL-6 levels from noninfected $(n=6)$ and chronic SIV-infected $(n=12)$ RMs. (C) IL-6Ra expression on CD4 T cell subsets from noninfected $(n=10)$, acute $(n=11)$, and chronic SIV-infected $(n=18)$ LNs. (D) Linear regression analysis between IL-6Ra and frequency of TFH cells (chronic SIV-infected, $n=18$ ). (E) IL-6-induced pSTAT-1 (3), after subtraction of basal (no stimulation) levels, in CD4 T cells from noninfected $(n=4)$, acute $(n=4)$, and chronic SIV-infected $(n=11)$ LNs. (F) Linear regression analysis between pSTAT-1 (pSTAT-1/pSTAT-3) in PD- $1^{\text {hi }}$ CD4 T cells and frequency of TFH cells (chronic SIV-infected RMs, $n=11$ ). (G) Viability and absolute cell numbers (counts after treatment minus initial counts) of sorted LN CD4 T cells (chronic SIV-infected, $n=4)$ stimulated with anti-CD3 with or without IL-6. (H) Pooled data showing levels of BCL-6 in LN $(n=5)$ CD4 T cell populations stimulated with anti-CD3 with or without IL-6. Box sizes represent limits of the second and third quartiles, with medians shown as bars. Whiskers define minimum and maximum of data. Each dot represents data for a single sample from an individual animal. Animals are represented once in each plot. Horizontal lines depict mean values. $P$ values were calculated using the $(\mathbf{A}-\mathbf{C})$ Mann-Whitney $U$ test or $(\mathbf{E})$ Wilcoxon matched-pairs signed-rank test. 
and CD127-PE (R34.34) (both from Beckman Coulter); CCR7-Pacific Blue (TG8/CCR7), CD150-PE (A12), ICOS-Alexa Fluor 647 (C398.4A), CD20 BV570 (catalog no. 302331), and CD20-APC (catalog no. 302310) (all from Biolegend); and CD4-Cy55PE (S3.5) and CD8-Qdot705 655 (3B5) (both from Invitrogen). IgD-FITC (catalog no. 2030-02) was obtained from SouthernBiotech, biotinylated anti-PD-1 antibody was obtained from R\&D Systems (BAF 1086), streptavidin-Cy7PE(Qdot 655) was obtained from Molecular Probes, and quantum dots were obtained from Quantum Dot Corporation. Aqua amine viability dye was purchased from Invitrogen, and PNA-PE was purchased from GeneTex.

\section{Flow cytometry}

$2 \times 10^{6}$ to $3 \times 10^{6}$ cells were stained with Aqua and aPD-1-biotin. Following a staining step with streptavidin, cells were surface stained with titrated amounts of aCD3, aCD4, aCD8, aCD28, aCD95, aICOS, aCD150, aCCR7, and aCXCR4 and fixed/permeabilized and stained with an aCTLA4. IL-6Ra, GP130, BTLA, CD127, CD69, and PD-L1 were also analyzed. Intracellular levels of CD154 were investigated after stimulation with SEB (Sigma-Aldrich) $(1 \mu \mathrm{g} / \mathrm{ml})$ at different time points.

TFH cell-BCL-6 staining. Surface stained cells were lysed with $100 \mu \mathrm{l} \mathrm{FACS}$ Lysing Solution (BD Pharmingen, catalog no. 349202), stained with antiBCL-6-PE (BD Pharmingen, catalog no. 561522) in the presence of $0.25 \%$ Saponin, and fixed with paraformaldehyde.

Detection of PSTAT-1/PSTAT-3. After staining with Aqua, CD4, and PD-1, cells were washed and incubated in RPMI plus $1 \%$ FCS for 10 minutes at $37^{\circ} \mathrm{C}, 5 \% \mathrm{CO}_{2}$. Cells were incubated with $40 \mathrm{ng} / \mathrm{ml}$ human IL- 6 (R\&D Systems, catalog no. 206-IL/CF) for 15 minutes (39) at $37^{\circ} \mathrm{C}, 5 \% \mathrm{CO}_{2}$ and immediately fixed with $4 \%$ paraformaldehyde. After centrifugation, cells were resuspended in $500 \mu \mathrm{l}$ Perm Buffer III (BD Biosciences, catalog no. 558050) and incubated for 30 minutes on ice. Cells were washed with Perm/Wash Buffer (BD Biosciences) and incubated with anti-STAT-3 and anti-STAT-1.

$B$ cell assays. Cells were stained with Aqua and $\mathrm{Fc}$ blocker (not used in the presence of anti-IgG) (Miltenyi Biotech, catalog no. 120-000-442), followed by a surface staining step with CD20/CD3/IgD/PD-L1/CD27 or CD20/ $\mathrm{CD} 3 / \mathrm{CD} 27 / \mathrm{IgG} / \mathrm{PNA}$ combinations. In some experiments, intracellular Ki67 staining was performed. BCL-6 staining was performed as in the case of TFH cells: surface staining (Aqua/CD3/CD20/IgD/IgG) was followed by intracellular staining for BCL-6 and Ki67. Cell populations were sorted under sterile conditions by using a FACS Aria system in a BSL-3 facility.

\section{Antibody measurements}

SIV-specific humoral IgG and IgA levels were evaluated by a standardized binding antibody multiplex assay, as previously described $(55,58)$. For IgA measurements, serum was depleted of IgG using protein $\mathrm{G}$ highperformance MultiTrap plates (GE Inc.), according to the manufacturer's instructions with minor modifications as previously described (55). SIV $_{\text {mac239 }}$ p55 GAG (Protein Sciences), SIV p27 GAG (ImmunoDiagnostics), rgp41 (Immunodiagnostics), SIV mac251 $_{1}$ rgp130 (ImmunoDiagnostics), and SIV gp140 (provided by Bing Chen, Harvard Medical School, Boston, Massachusetts, USA) proteins were coupled to microspheres (Bio-Rad) and incubated with serum. For IgG antibodies, serial dilutions of samples starting at a 1:80 dilution, each in duplicate, were detected by biotinylated anti-monkey IgG (Rockland), and serum titers for SIV IgG were calculated by area under the curve (GraphPad Prism). IgA antibodies were detected by biotinylated anti-IgA (Jackson ImmunoResearch Inc.), with demonstrated lack of cross-reactivity to macaque IgG proteins (provided by Keith A. Reimann, Beth Israel Deaconess Medical School, Boston, Massachusetts, USA). IgG-depleted serum was tested at 1:80 final dilution, and SIV-specific IgA responses were reported as MFI within the linear range of the assay, with background subtracted (plate and blank bead). DBM5 SIV-positive serum control (midpoint titer [ $\left.\mathrm{EC}_{50}\right], 4-\mathrm{PL}$ fit) was included in each assay and met preset criteria to track antigen performance (Levey-Jennings plot).

Surface plasmon resonance assay. Surface plasmon resonance binding avidity measurements were performed on the BIAcore 3000 (GE Healthcare/ BIAcore) with purified serum IgG. SIV antigens were anchored on duplicate spots using standard amine coupling chemistry on a BIAcore $\mathrm{S}$ series CM5 sensor chip. SIV-positive macaque sera (DBM5) were used as control and anti-RSV mAb Synagis was used as a negative control to subtract nonspecific binding to the sensor surface. Maximal binding response at the end of the injection phase ( 5 minutes) and dissociation rate constants $\left(\mathrm{k}_{\mathrm{d}}, \mathrm{s}^{-1}\right)$ were calculated from each binding curve. Relative binding avidity scores were calculated (avidity score $[\mathrm{RUs}]=\left[\right.$ binding response units $\left./ \mathrm{k}_{\mathrm{d}}\right]$ ) and expressed as RU $\times \mathrm{s}$, with higher binding responses and slower dissociation constant $\mathrm{k}_{\mathrm{d}}$ as an indicator of higher affinity interaction.

\section{Imaging studies}

6- to $10-\mu \mathrm{m}$ formalin-fixed, paraffin-embedded sections were deparaffinized, and antigen retrieval was performed by 30 -minute incubation in $0.1 \mathrm{M}$ Sodium Citrate EDTA buffer ( $\mathrm{pH} 6.2$ ) at $100^{\circ} \mathrm{C}$. Following incubation with Image-IT FX signal enhancer (Invitrogen) and blocking for 1 hour $(0.1 \mathrm{M}$ Tris, $0.3 \%$ Triton X-100, 1\% bovine serum albumin, $1 \%$ human), sections were sequentially stained with aPD-1 (R\&D Systems, catalog no. AF1086), aCD20cy (Dako, L26), CD4 (Leica, 1F6), and Ki67Alexa Fluor 700 (eBioscience, B56). Slides were imaged with a Zeiss 710 confocal microscope using a $\times 200.8$ NA objective, with a $\times 2$ optical zoom, or with a Leica SP5 confocal microscope using a $\times 401.25$ NA objective, with a $\times 1.5$ optical zoom, at a 1,024 $\times 1,024$ pixel density. All image analysis was performed with Imaris software (Bitplane Scientific Software) in combination with Excel (Microsoft) and Prism (GraphPad). Nonspecific signal from autofluorescent macrophages was masked out of the final image analysis using a dedicated autofluorescence channel and non-autofluorescent region of interest selection. Presented images are maximum projections of 3 to 5 separate confocal Z slices. Standard 3-dimensional generated surfaces, representing either whole CD4 T cells or portions of CD4 $\mathrm{T}$ cell plasma membranes, and watershed segmentation algorithms in Imaris were applied to the CD4 signal. Average pixel intensity of the $\mathrm{PD}-1$ stain inside each $\mathrm{CD} 4$ surface, along with the $\mathrm{X}, \mathrm{Y}$ positioning of the surface center, was exported to Excel. Distance to the center of the B cell follicle of each surface was determined.

\section{Microarray and pathway analysis}

Cells from SIV-negative (non-TFH, $n=5 ; \mathrm{TFH}, n=5$ ) and chronic SIVinfected $(n=6)$ SP tissues were sorted for microarray studies. RNA samples were prepared using the Illumina beads station assay, as previously described $(59,60)$, and hybridized to the Illumina HumanHT-12 version 4 Expression BeadChip, according to the Illumina's instruction. The data were normalized with the quantile normalization method of Bioconductor package limma (61). Missing values were imputed with R package impute (http://www.bioconductor.org/packages/ release/bioc/html/impute.html). Genes with significant differential expression levels were identified using Bioconductor package limma with 1.5 or higher fold change (up or down), and the false discovery rate adjusted the raw $P$ value of less than 0.05 . The top 200 genes for the 2 contrasts were selected to generate heat maps using $\mathrm{R}$ package pheatmap (http://cran.r-project.org/web/packages/pheatmap/index. html). Network analysis was done with Ingenuity Pathway Analysis software (Ingenuity Systems). The microarray data has been deposited at http://www.ncbi.nlm.nih.gov/geo/query/acc.cgi?acc=GSE38795 (GEO accession no. GSE38795). 
A
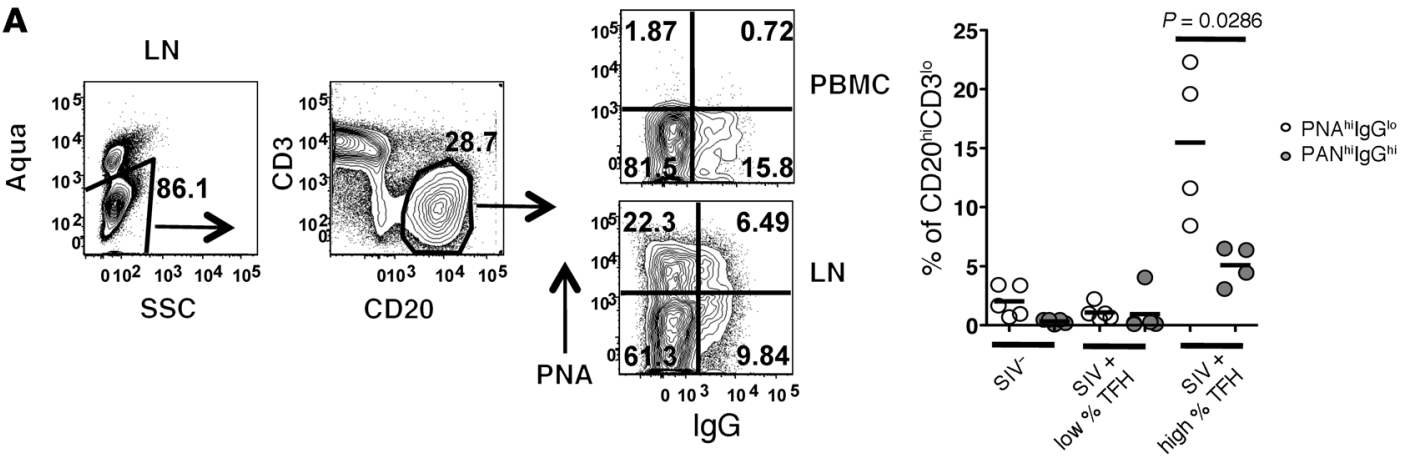

B LN: CD20 ${ }^{\text {hi }} \mathrm{CD}^{\text {lo }}$
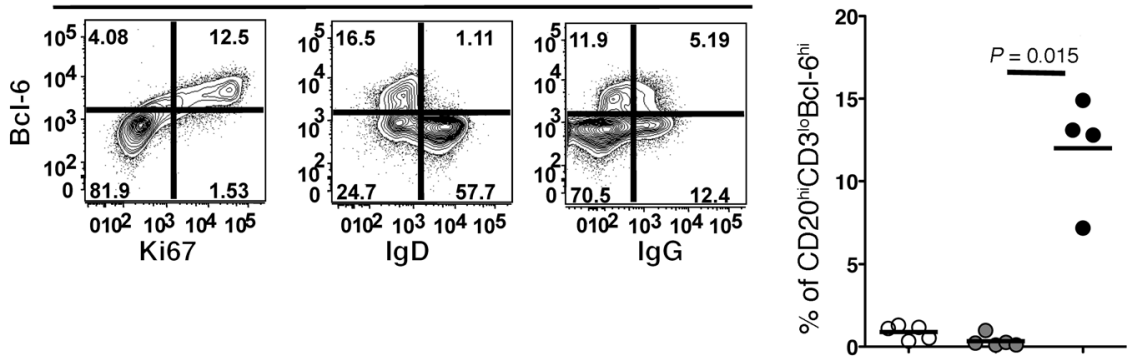

OSIV-

- SIV chronic (low \% TFH)

- SIV chronic (high \% TFH)

$\lg \mathrm{G}$
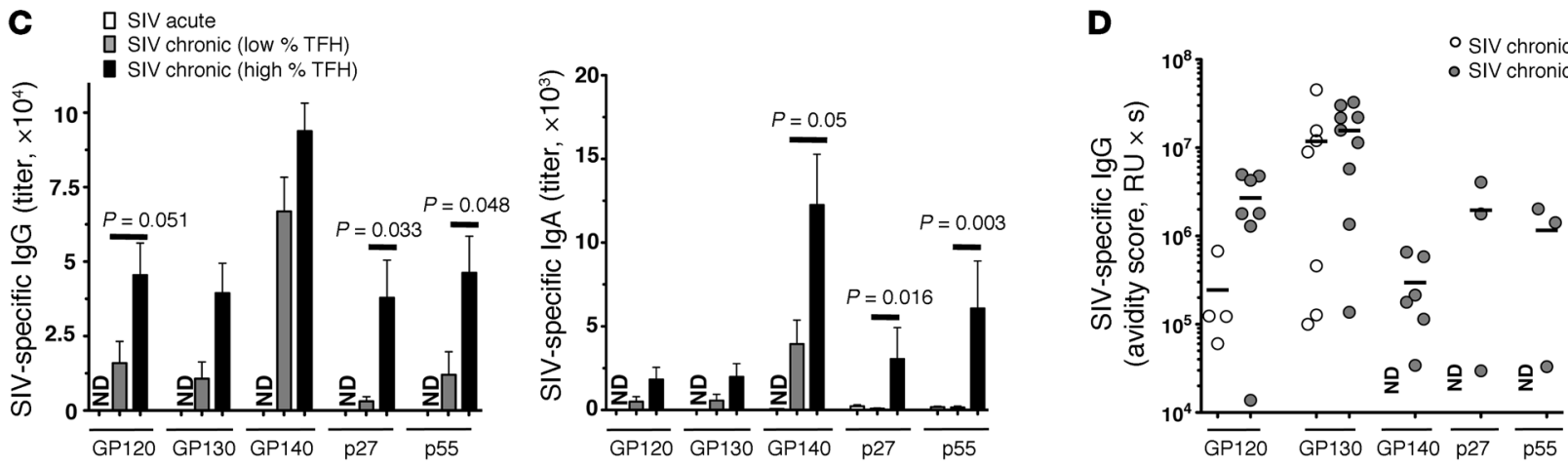

Figure 7

Accumulation of TFH cells in chronic SIV infection is associated with increased generation of GC B cells and SIV-specific antibodies. (A) Flow cytometry plots showing the binding of PNA within the B cell compartment in the LNs and PBMCs from a chronic SIV-infected RM as well as pooled data showing the frequency $(\%)$ of PNA ${ }^{\text {hilgG }}{ }^{\text {hi }}$ and PNA hilgGlo B cells in LNs from noninfected $(n=5)$ and chronic SIV-infected RMs ( $n=5$ for low percentage of TFH LNs and $n=4$ for high percentage TFH LNs). (B) Contour plots showing the coexpression of BCL-6 and Ki67 or IgD or IgG in B cells from a chronic SIV-infected LN as well as pooled data showing the frequency (\%) of BCL-6 $6^{\text {hi }} B$ cells in LNs from noninfected $(n=5)$ and chronic SIV-infected RMs ( $n=5$ for low percentage of TFH LNs and $n=4$ for high percentage TFH LNs). (C) Pooled data showing the IgG and IgA titers of SIV-specific antibodies in plasma from acute SIV-infected ( $n=7$; white symbols) and chronic SIV-infected RMs ( $n=7$ for low percentage of TFH cells, gray symbols; $n=9$ for high percentage TFH cells, black symbols). (D) Pooled data showing the avidity score for plasma purified IgG from chronic SIV-infected RMs. The frequency of the gated populations is shown in the contour plots. Horizontal lines depict mean values. $P$ values were calculated using Mann-Whitney $U$ test.

\section{qRT and DNA PCR studies}

mRNA was analyzed using the SuperScript III Platinum One-Step qRTPCR system (Invitrogen), and relative amounts were calculated by the comparative $(\Delta \Delta)$ Ct method. The following primers and probes were used: BCL6 (Hs00277037_m1), IL21 (Hs00222327_m1), MAF(Hs00193519_m1), GAPDH (Hs99999905_m1), TBX21 (also known as T-bet) (Rh02621772_ m1), CXCL13 (Hs00757930_m1), and S1PR1 (Hs00173499_m1). All primers were from Applied Biosystems, Life Technologies. SIV-GAG DNA in sorted cells was measured as previously described (62).

\section{Cytokine detection}

Sorted cells were stimulated with PMA $(10 \mathrm{ng} / \mathrm{ml}) /$ ionomycin $(1 \mu \mathrm{g} / \mathrm{ml})$ for 14-16 hours, and supernatants were collected and subjected to cytokine analysis using the Luminex technology according to the manufacture's instructions (Milliplex Kit, catalog no. MPXPRCYTO-40K, Milipore).

\section{In vivo administration of $B r d U$}

BrdU (Sigma-Aldrich, B5002-5G) was dissolved in Hanks Balanced Salt Solution (Invitrogen) at $10 \mathrm{mg} / \mathrm{ml}$ (pH 7.4) and sterile filtered into auto- 
claved bottles. Chronic SIV-infected RMs $(n=3)$ received $30 \mathrm{mg} / \mathrm{kg}$ body weight of BrdU via intravenous injection daily on 4 consecutive days. LN tissues (ipsilateral or contralateral to the site of the BrdU injection) were collected on day $3,8,13$, and 20 after the initiation of BrdU administration.

\section{Plasma viral load}

Levels of plasma SIV were determined by quantitative RT-PCR as previously described (63).

\section{Measurement of sCD14 and IL-6 levels}

sCD14 and IL- 6 levels were determined in plasma by ELISA according to the manufacturer's instructions (R\&D Systems, catalog no. DC140 for sCD14 and catalog no. D6050 for IL-6).

\section{In vitro assays}

Cell death. Fresh cells were cultured for 14 to 16 hours, harvested, and surface stained with Aqua, aCD3-Cy7APC, aCD4-Cy55PE, aCD28-TRPE, aCD95Cy5PE, aCCR7-Pacific Blue, aPD-1-biotin, and aCD150-PE. Intracellular staining was performed using an antibody against active caspase-3-FITC.

Proliferation and cell cycle analysis. Freshly sorted cells from chronic SIVinfected LNs were cultured for 4 to 5 days in the presence of plate-bound anti-CD3 $(5 \mu \mathrm{g} / \mathrm{ml})$ (Cell Science, FN18) and in the absence or presence of IL-6 $(40 \mathrm{ng} / \mathrm{ml})$. The amount of the in vitro cell death was calculated based on the Aqua binding. The absolute cell numbers were counted using a Cellometer Vision system (Nexselom Biosciences). Cells were incubated with BrdU (BD, catalog no. 552598) for 30 minutes, harvested, stained with Aqua, and treated with FACS Lysing Solution (BD Pharmingen, catalog no. 349202). Following a fixed/permeabilization step (BD), cells were treated for 30 minutes with DNAse and stained with aKi67-FITC, aBrdU-APC (BD catalog no. 552598), and 7AAD.

Tand B cell coculture. $10^{5}$ to $5 \times 10^{5} \mathrm{LN}$ CD $4 \mathrm{~T}$ cell populations were sorted based on the expression of PD-1 and cocultured with autologous sorted LN B cells at a 1:1 ratio in the presence of SEB $(2 \mu \mathrm{g} / \mathrm{ml})$ for 12 days. Supernatants were harvested and analyzed for total $\operatorname{IgG}$ with a commercially available ELISA Kit (Alpha Diagnostic International).

Effect of IL-6 on TFH-related proteins. LN cell suspensions $\left(10^{6}\right.$ cells/well) were cultured with medium or medium plus IL-6 $(40 \mathrm{ng} / \mathrm{ml})$ in the absence or presence of immobilized anti-CD3 (FN18) $(5 \mu \mathrm{g} / \mathrm{ml})$. Cells were har- vested after 24 and 72 hours, and the levels of BCL-6, ICOS, CD95, and PD-1 were analyzed with a flow cytometry assay.

\section{Statistics}

Experimental variables were analyzed using the nonparametric MannWhitney test or the Wilcoxon matched-pairs signed-rank test. Linear regression analysis was applied for certain variables. When box-and-whiskers plots are used, the box size represents the limits of the data for the second and third quartiles, with median shown as a bar. The whiskers define the minimum and maximum of the data presented. Bars depict mean + SEM in all bar graphs in which error bars are shown. The GraphPad Prism statistical analysis program (GraphPad Software) was used throughout. $P$ values of less than 0.05 were considered significant.

\section{Study approval}

The described studies were under protocols reviewed and approved by the institutional animal care and use committee of the Vaccine Research Center.

\section{Acknowledgments}

The authors would like to thank the personnel of the Flow Cytometry Core, the Nonhuman Primate Immunogenicity Core Section, and the Laboratory Animal Medicine at Vaccine Research Center as well as the Aids Vaccine Program, SAIC-Frederick Inc., NCI-Frederick, and R. Glenn Overman, Will Williams, and Shelley Stewart at Duke Human Vaccine Institute. We would like to thank Adrian B. McDermott and Brenna Hill for helpful discussions. This research was supported by the Intramural Research Program of the Vaccine Research Center and a CAVD grant (no. OPP1032325) from the Bill and Melinda Gates Foundation (to R.A. Koup).

Received for publication April 27, 2012, and accepted in revised form July 5, 2012.

Address correspondence to: Constantinos Petrovas, Immunology Laboratory, Vaccine Research Center, NIAID, NIH, 40 Convent Drive, MSC 3022, Building 40, Room 3612B, Bethesda, Maryland 20892, USA. Phone: 301.594.8573; Fax: 301.480.2779; E-mail: petrovasc@mail.nih.gov.
1. Crotty S. Follicular helper CD4 T cells (TFH). Annu Rev Immunol. 2010;29:621-663.

2. Nutt SL, Tarlinton DM. Germinal center B and follicular helper T cells: siblings, cousins or just good friends? Nat Immunol. 2011;12(6):472-477.

3. McHeyzer-Williams LJ, Pelletier N, Mark L, Fazilleau N, McHeyzer-Williams MG. Follicular helper $\mathrm{T}$ cells as cognate regulators of B cell immunity. Curr Opin Immunol. 2009;21(3):266-273.

4. Yu D, Vinuesa CG. The elusive identity of T follicular helper cells. Trends Immunol. 2010;31(10):377-383.

5. Deenick EK, Ma CS, Brink R, Tangye SG. Regulation of $\mathrm{T}$ follicular helper cell formation and function by antigen presenting cells. Curr Opin Immunol. 2011;23(1):111-118.

6. Haynes NM, Allen CD, Lesley R, Ansel KM, Killeen N, Cyster JG. Role of CXCR5 and CCR7 in follicular Th cell positioning and appearance of a programmed cell death gene-1high germinal center-associated subpopulation. J Immunol. 2007; 179(8):5099-5108.

7. Nurieva RI, et al. Bcl6 mediates the development of T follicular helper cells. Science. 2009; 325(5943):1001-1005.

8. Yu D, et al. The transcriptional repressor Bcl-6 directs $\mathrm{T}$ follicular helper cell lineage commitment. Immunity. 2009;31(3):457-468.
9. Fazilleau N, McHeyzer-Williams LJ, Rosen H, McHeyzer-Williams MG. The function of follicular helper $\mathrm{T}$ cells is regulated by the strength of $\mathrm{T}$ cell antigen receptor binding. Nat Immunol. 2009;10(4):375-384.

10. Goenka R, et al. Cutting edge: dendritic cellrestricted antigen presentation initiates the follicular helper $\mathrm{T}$ cell program but cannot complete ultimate effector differentiation. J Immunol. 2011;187(3):1091-1095

11. Hardtke S, Ohl L, Förster R. Balanced expression of CXCR5 and CCR7 on follicular T helper cells determines their transient positioning to lymph node follicles and is essential for efficient B-cell help. Blood. 2005;106(6):1924-1931.

12. Choi YS, et al. ICOS receptor instructs $T$ follicular helper cell versus effector cell differentiation via induction of the transcriptional repressor Bcl6. Immunity. 2011;34(6):932-946.

13. Cannons JL, et al. SAP regulates $\mathrm{T}(\mathrm{H}) 2$ differentiation and PKC-theta-mediated activation of NFkappaB1. Immunity. 2004;21(5):693-706.

14. Qi H, Cannons JL, Klauschen F, Schwartzberg PL, Germain RN. SAP-controlled T-B cell interactions underlie germinal centre formation. Nature. 2008;455(7214):764-769.

15. Chtanova $\mathrm{T}$, et al. $\mathrm{T}$ follicular helper cells express a distinctive transcriptional profile, reflecting their role as non-Th1/Th2 effector cells that provide help for B cells. J Immunol. 2004;173(1):68-78.

16. Kim CH, Lim HW, Kim JR, Rott L, Hillsamer P, Butcher EC. Unique gene expression program of human germinal center T helper cells. Blood. 2004;104(7):1952-1960.

17. Kim CH, Rott LS, Clark-Lewis I, Campbell DJ, Wu L, Butcher EC. Subspecialization of CXCR5+ $\mathrm{T}$ cells: B helper activity is focused in a germinal center-localized subset of CXCR5 + T cells. J Exp Med. 20018;193(12):1373-1381.

18. Ma CS, et al. Early commitment of naive human $\mathrm{CD} 4(+) \mathrm{T}$ cells to the $\mathrm{T}$ follicular helper $(\mathrm{T}(\mathrm{FH}))$ cell lineage is induced by IL-12. Immunol Cell Biol. 2009;87(8):590-600.

19. Fahey LM, Wilson EB, Elsaesser H, Fistonich CD, McGavern DB, Brooks DG. Viral persistence redirects $\mathrm{CD} 4 \mathrm{~T}$ cell differentiation toward $\mathrm{T}$ follicular helper cells. J Exp Med. 2011;208(5):987-999.

20. Zaretsky AG, Taylor JJ, King IL, Marshall FA, Mohrs $\mathrm{M}$, Pearce EJ. T follicular helper cells differentiate from Th2 cells in response to helminth antigens. J Exp Med. 2009;206(5):991-999.

21. Nurieva RI, et al. Generation of T follicular helper cells is mediated by interleukin-21 but independent of T helper 1, 2, or 17 cell lineages. Immunity. 
2008;29(1):138-149.

22. Harker JA, Lewis GM, Mack L, Zuniga EI. Late interleukin- 6 escalates $T$ follicular helper cell responses and controls a chronic viral infection. Science. 2011;334(6057):825-829.

23. Pepper M, Jenkins MK. Origins of CD4(+) effector and central memory $\mathrm{T}$ cells. Nat Immunol. 2011;12(6):467-471.

24. Hong JJ, Amancha PK, Rogers K, Ansari AA, Villinger F. Spatial alterations between CD4(+) T follicular helper, B, and CD8(+) T cells during simian immunodeficiency virus infection: $\mathrm{T} / \mathrm{B}$ cell homeostasis, activation, and potential mechanism for viral escape. J Immunol. 2012;188(7):3247-3256.

25. Pitcher CJ, et al. Development and homeostasis of $\mathrm{T}$ cell memory in rhesus macaque. J Immunol. 2002;168(1):29-43.

26. Letvin NL, et al. Immune and genetic correlates of vaccine protection against mucosal infection by SIV in monkeys. Sci Transl Med. 2011;3(81):81ra36.

27. Rayman N, et al. Distinct expression profiles of the peripheral cannabinoid receptor in lymphoid tissues depending on receptor activation status. J Immunol. 2004;172(4):2111-2117.

28. Bauquet AT, et al. The costimulatory molecule ICOS regulates the expression of c-Maf and IL-21 in the development of follicular T helper cells and TH-17 cells. Nat Immunol. 2009;10(2):167-175.

29. Ise W, et al. The transcription factor BATF controls the global regulators of class-switch recombination in both B cells and T cells. Nat Immunol. 2011;12(6):536-543.

30. Morita R, et al. Human blood CXCR5 $(+)$ CD4(+) $\mathrm{T}$ cells are counterparts of $\mathrm{T}$ follicular cells and contain specific subsets that differentially support antibody secretion. Immunity. 2011;34(1):108-121.

31. O'Keeffe M, et al. Effects of administration of progenipoietin 1, Flt-3 ligand, granulocyte colonystimulating factor, and pegylated granulocyte-macrophage colony-stimulating factor on dendritic cell subsets in mice. Blood. 2002;99(6):2122-2130.

32. Linterman MA, et al. Foxp3+ follicular regulatory $\mathrm{T}$ cells control the germinal center response. Nat Med. 2011;17(8):975-982.

33. Good-Jacobson KL, Szumilas CG, Chen L, Sharpe $\mathrm{AH}$, Tomayko MM, Shlomchik MJ. PD-1 regulates germinal center B cell survival and the formation and affinity of long-lived plasma cells. Nat Immunol. 2010;11(6):535-542.

34. Schwab SR, Cyster JG. Finding a way out: lymphocyte egress from lymphoid organs. Nat Immunol. 2007;8(12):1295-1301.

35. Chakrabarti LA, et al. Normal T-cell turnover in sooty mangabeys harboring active simian immunodeficiency virus infection. J Virol. 2000;74(3):1209-1223.

36. Sandler NG, et al. Plasma levels of soluble CD14 independently predict mortality in HIV infection. J Infect Dis. 2011;203(6):780-790.

37. Feng J, et al. High frequency of CD4+ CXCR5+ TFH cells in patients with immune-active chronic hepatitis B. PLoS One. 2011;6(7):e21698.

38. Kuller LH, et al. Inflammatory and coagulation biomarkers and mortality in patients with HIV infection. PLoS Med. 2008;5(10):e203.

39. Zhang X, Blenis J, Li HC, Schindler C, Chen-Kiang $\mathrm{S}$. Requirement of serine phosphorylation for formation of STAT-promoter complexes. Science. 1995;267(5206):1990-1994.

40. Yu CR, Young HA, Ortaldo JR. Characterization of cytokine differential induction of STAT complexes in primary human T and NK cells. J Leukoc Biol. 1998;64(2):245-258.

41. Ma J, Ma D, Ji C. The role of IL-21 in hematological malignancies. Cytokine. 2011;56(2):133-139.

42. Vogelzang A, McGuire HM, Yu D, Sprent J, Mackay CR, King C. A fundamental role for interleukin-21 in the generation of $\mathrm{T}$ follicular helper cells. Immunity. 2008;29(1):127-137.

43. Breitfeld D, et al. Follicular B helper T cells express CXC chemokine receptor 5, localize to B cell follicles, and support immunoglobulin production. J Exp Med. 2000;192(11):1545-1552.

44. Cannons JL, Tangye SG, Schwartzberg PL. SLAM family receptors and SAP adaptors in immunity. Annu Rev Immunol. 2011;29:665-705.

45. Yusuf I, et al. Germinal center T follicular helper cell IL-4 production is dependent on signaling lymphocytic activation molecule receptor (CD150). J Immunol. 2010;185(1):190-202.

46. Cannons JL, et al. Biochemical and genetic evidence for a SAP-PKC-theta interaction contributing to IL-4 regulation. Jimmunol. 2010;185(5):2819-2827.

47. Zeng M, et al. Cumulative mechanisms of lymphoid tissue fibrosis and T cell depletion in HIV-1 and SIV infections. J Clin Invest. 2011;121(3):998-1008.

48. Wang $\mathrm{H}$, et al. C/EBPalpha arrests cell proliferation through direct inhibition of Cdk2 and Cdk4. Mol Cell. 2001;8(4):817-828.

49. Lopez RG, et al. C/EBPalpha and beta couple interfollicular keratinocyte proliferation arrest to commitment and terminal differentiation. Nat Cell Biol. 2009;11(10):1181-1190

50. Porse BT, et al. Loss of C/EBP alpha cell cycle control increases myeloid progenitor proliferation and transforms the neutrophil granulocyte lineage. J Exp Med. 2005;202(1):85-96.
51. Zhang P, Iwama A, Datta MW, Darlington GJ, Link DC, Tenen DG. Upregulation of interleukin 6 and granulocyte colony-stimulating factor receptors by transcription factor CCAAT enhancer binding protein alpha (C/EBP alpha) is critical for granulopoiesis. J Exp Med. 1998;188(6):1173-1184.

52. Mackey SL, Darlington GJ. CCAAT enhancerbinding protein alpha is required for interleukin- 6 receptor alpha signaling in newborn hepatocytes. J Biol Chem. 2004;279(16):16206-16213.

53. Müller C, Alunni-Fabbroni M, Kowenz-Leutz E, Mo X, Tommasino M, Leutz A. Separation of C/ EBPalpha-mediated proliferation arrest and differentiation pathways. Proc Natl Acad Sci U S A. 1999;96(13):7276-7281.

54. Hu X, Ivashkiv LB. Cross-regulation of signaling pathways by interferon-gamma: implications for immune responses and autoimmune diseases. Immunity. 2009;31(4):539-550

55. Tomaras GD, et al. Initial B-cell responses to transmitted human immunodeficiency virus type 1 : virion-binding immunoglobulin $\mathrm{M}(\mathrm{IgM})$ and $\operatorname{IgG}$ antibodies followed by plasma anti-gp41 antibodies with ineffective control of initial viremia.J Virol. 2008;82(24):12449-12463.

56. Mattapallil JJ, et al. Vaccination preserves CD4 memory $\mathrm{T}$ cells during acute simian immunodeficiency virus challenge. J Exp Med. 2006;203(6):1533-1541.

57. Mattapallil JJ, Douek DC, Hill B, Nishimura Y, Martin $\mathrm{M}$, Roederer M. Massive infection and loss of memory CD4+ T cells in multiple tissues during acute SIV infection. Nature. 2005;434(7037):1093-1097.

58 . Bolton DL, et al. Comparison of systemic and mucosal vaccination: impact on intravenous and rectal SIV challenge. Mucosal Immunol. 2012;5(1):41-52.

59. Quakkelaar ED, et al. Improved innate and adaptive immunostimulation by genetically modified HIV-1 protein expressing NYVAC vectors. PLoS One. 2011;6(2):e16819.

60 . Gaucher D, et al. Yellow fever vaccine induces integrated multilineage and polyfunctional immune responses. J Exp Med. 2008;205(13):3119-3131.

61. Smyth GK. Linear models and empirical bayes methods for assessing differential expression in microarray experiments. Stat Appl Genet Mol Biol. 2004;3:Article3.

62. Douek DC, et al. HIV preferentially infects HIV-specific CD4+ T cells. Nature. 2002;417(6884):95-98.

63. Cline AN, Bess JW, Piatak M Jr, Lifson JD. Highly sensitive SIV plasma viral load assay: practical considerations, realistic performance expectations, and application to reverse engineering of vaccines for AIDS. J Med Primatol. 2005;34(5-6):303-312. 